\title{
A Discrete Model for an Ill-posed Nonlinear Parabolic PDE
}

\author{
Thomas P. Witelski ${ }^{a}$, David G. Schaeffer ${ }^{a}$ and \\ Michael Shearer ${ }^{b}$ \\ ${ }^{\mathrm{a}}$ Department of Mathematics and the Center for Nonlinear and Complex Systems, \\ Duke University, Durham, NC 27708-0320, witelski@math.duke.edu, \\ dgs@math.duke.edu \\ ${ }^{\mathrm{b}}$ Department of Mathematics and the Center for Research in Scientific \\ Computation, North Carolina State University, Raleigh, NC 27695-8205, \\ shearer@math.ncsu.edu
}

\begin{abstract}
We study a finite-difference discretization of an ill-posed nonlinear parabolic partial differential equation. The PDE is the one-dimensional version of a simplified two-dimensional model for the formation of shear bands via anti-plane shear of a granular medium. For the discretized initial value problem, we derive analytically, and observed numerically, a two-stage evolution leading to a steady-state: (i) an initial growth of grid-scale instabilities, and (ii) coarsening dynamics. Elaborating the second phase, at any fixed time the solution has a piecewise linear profile with a finite number of shear bands. In this coarsening phase, one shear band after another collapses until a steady-state with just one jump discontinuity is achieved. The amplitude of this steady-state shear band is derived analytically, but due to the ill-posedness of the underlying problem, its position exhibits sensitive dependence. Analyzing data from the simulations, we observe that the number of shear bands at time $t$ decays like $t^{-1 / 3}$. From this scaling law we show that the time-scale of the coarsening phase in the evolution of this model for granular media critically depends on the discreteness of the model.

Our analysis also has implications to related ill-posed nonlinear PDEs for the onedimensional Perona-Malik equation in image processing and to models for clustering instabilities in granular materials.
\end{abstract}

Key words: nonlinear PDE, illposed equations, nonlinear diffusion, granular medium, shear bands 


\section{Introduction}

The degenerate parabolic PDE,

$$
\frac{\partial v}{\partial t}=\operatorname{div}\left(R \frac{\nabla v}{|\nabla v|}\right)
$$

in which $R$ is a rotation matrix, arises from a simplified model of the velocity field of a sheared granular material [33]. This equation is ill-posed, a property typical of continuum models of granular media $[25,31,32,34]$. To study the dynamics of this model, in this paper we analyze finite difference approximations of the PDE (1.1). More precisely, we discretize in space, and study the resulting system of ODEs, which we refer to as the discrete model. This model is a form of regularization of (1.1); the discrete model is well-posed, mollifying instabilities at the highest frequencies.

The simulations show that amplification of perturbations at short wavelengths lead quickly to small-amplitude discontinuities, see Figure 1b. This short time behavior is followed by a gradual coarsening and long time evolution to a steady state. The steady state has a single discontinuity that we refer to as a shear band [9], see Figure 1c. The time-scale for the coarsening dynamics phase of the evolution diverges as the mesh scale $\Delta x$ tends to zero. Thus, strictly speaking, in the continuum limit, no evolution occurs! In other words, the discreteness of the finite difference model is essential to the dynamics of this system. Implications of this result for granular flows will be discussed further in a subsequent paper.

The behavior of this nonlinear ill-posed equation should be contrasted with the behavior of ill-posed linear equations where short wavelength disturbances are simply amplified catastrophically. Correspondingly, solutions of difference approximations of linear ill-posed equations diverge in every norm, as the mesh spacing approaches zero. For our discrete model of (1.1), as $\Delta x \rightarrow 0$, the sequence of solutions of the difference equations converges in $L^{2}$, but because of ill-posedness it blows-up in $H^{1}$. The divergence in $H^{1}$ is a consequence of the very rapid development of "infinitesimal discontinuities" in the solution. These observations on the dynamics in the system are fully developed in Section 6.

The linear ill-posedness of the time-dependent equations is related to the property that the steady-state equations are hyperbolic. The steady-state equations are well-known to support shock waves [22]. In nonlinear continuum descriptions of granular materials linear ill-posedness means that initial value problems are sensitive to small perturbations, but it also provides a mechanism for the formation of fine-scale localized structures such as shear bands and shocks.

PDE models for granular flow are the result of treating the material as a continuum, allowing dynamics at all wavelengths, whereas in fact wavelengths much shorter than the grain size of particles in the granular media have no physical significance. Regarding the finite difference mesh parameter as being on the order of the grain size, we effectively replace the continuum description by a discrete model that may more faithfully represent the range of wavelengths 

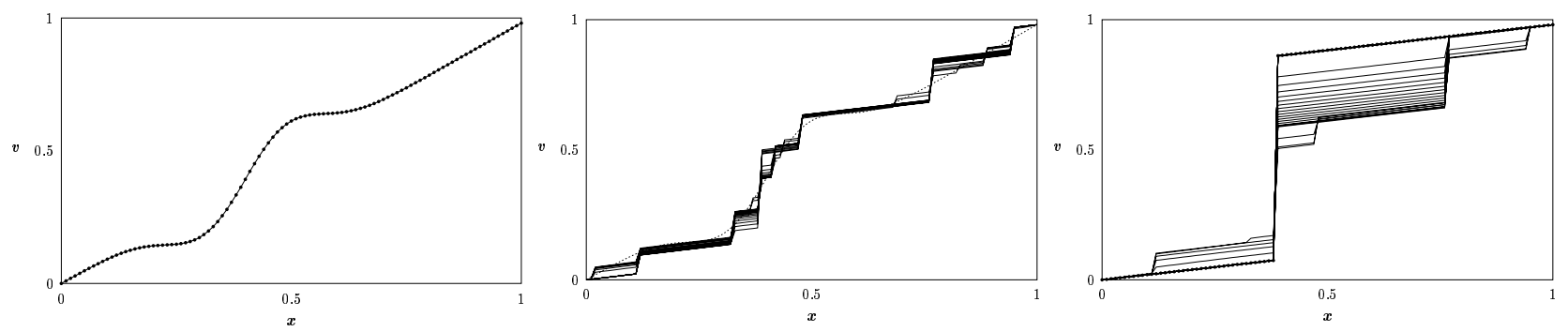

Fig. 1. One-dimensional evolution in (1.1); (a) initial data, (b) short time behavior, (c) long-time evolution to the steady-state shear band.

relevant for granular flow. The discrete model is analogous to discrete mechanical models whose continuum limit yields a continuum description, as has been used in constructing continuous models of high density discrete mechanical systems [26-28].

Ill-posed nonlinear parabolic equations also arise in population dynamics in mathematical biology [21,23], edge enhancement in image processing [2,5,8,20], and other problems in granular media flows [10,19,35]. Since the early work of Perona and Malik [24], ill-posed nonlinear diffusion equations have been used to produce enhancement of edges in digitized images by selective amplification of intensity gradients via backward diffusion. These models have attracted a lot of attention in the engineering and mathematical literature [20], and there are still many mathematical open questions. While (1.1) has a very different motivation than the Perona-Malik models, it shares many structural similarities, and some of our results may have further implications for image processing. Recent studies [10,35] of clustering instabilities (also called inelastic collapse) in dilute granular gases [19] have yielded models with non-monotone flux functions and discrete diffusive coupling in space. The discrete model considered here has some qualitative features similar to the clustering dynamics observed in those papers.

\subsection{Outline of paper}

The remainder of this paper is organized as follows. In Section 2 we formulate the PDE model in one and two space dimensions, and give preliminary information concerning the discrete model. Specifically, in Section 2.1, we discuss the PDE in the context of anti-plane shear, and specify the nature of the linear ill-posedness. In Section 2.2, we consider plane wave perturbations of uniform shear. We show that the resulting PDE in one space variable and time has the character of a backward-forward heat equation. In Section 2.3, we write down the discrete model, and show that solutions remain bounded globally in time, and uniformly in mesh spacing $\Delta x$.

In Section 3, we describe all equilibrium solutions of the discrete model. Apart from the trivial solution, corresponding to uniform shearing, equilibrium solutions have one or more finite jumps (that persist under mesh refinement), which we call shocks or shear bands. In Section 4 , we show that the trivial solution and single shear band solutions are the only 
possible stable equilibria. We characterize precise conditions under which these equilibria are in fact stable. Multiple shock solutions are shown to be unstable, a property that helps explain the coarsening exhibited in Figure 1. The proof is based on identifying a Liapunov function for the discrete model, and exploring the nature of it's critical points.

In Section 5, we describe detailed dynamic simulations like those of Figure 1. In Section 6, we discuss the continuum limit $\Delta x \rightarrow 0$, demonstrating the existence, via numerical experiments, of a scaling law for the evolution of shear bands in the coarsening process. Finally, in Section 7, we analyze the coarsening that occurs in the intermediate dynamics. Specifically, in Section 7.1, we analyze the coarsening from a solution with $K$ shocks to a solution with $K-1$ shocks, when $K$ is large. In Section 7.2, we formulate a reduced discrete model that describes the evolution of the shocks in isolation from the smooth part of the solution. The comparison of predictions from the reduced model and the full model helps to justify our explanation of how the coarsening process takes place.

\section{Formulation of the problem}

\subsection{The continuous two-dimensional model}

The dependent variable $v=v(x, y, t)$ in equation (1.1) may be thought of as the velocity in the $z$-direction in a block of material undergoing anti-plane shearing. The equation for conservation of momentum equates acceleration, $\partial_{t} v$, at constant density (normalized to unity) with the divergence of stress, $\nabla \cdot \vec{\tau}$. Modeling stress by the vector $\vec{\tau}=R \nabla v /|\nabla v|$, in which $R$ is the matrix representing a rotation counterclockwise through a constant angle $\alpha$,

$$
R=\left(\begin{array}{rr}
\cos \alpha & -\sin \alpha \\
\sin \alpha & \cos \alpha
\end{array}\right),
$$

with $0<\alpha<\pi / 2$, we arrive at equation (1.1). This model and related equations are studied in a series of papers $[3,4,6,13,12,25,31,32,34]$. Further details relating this constitutive relation to the full stress tensor and to properties of a perfectly plastic material are given in [33]. Equation (1.1) is a special case of the model in [33] subject to constant yield strength, specifically $|\tau|=1$.

The following proposition, adapted from [33], identifies the ill-posedness exhibited by (1.1). Consider a specific solution $v_{0}(x, y, t)$ of (1.1). If we linearize the equation about $v_{0}$ and freeze coefficients of the linearized equation at a point $\left(x_{0}, y_{0}, t_{0}\right)$, the resulting equation admits solutions in the form $\exp \left\{\lambda t+i\left(\xi_{1} x+\xi_{2} y\right)\right\}$. The equation relating $\lambda$ to $\vec{\xi}=\left(\xi_{1}, \xi_{2}\right)$ is the dispersion relation for the linear equation. For this equation, $\lambda$ is real, and we say the equation is ill-posed if $\lambda$ is not uniformly bounded above in $\vec{\xi}$. The proposition describes the nature of this ill-posedness, identifying a wedge of directions $\vec{\xi}$ within which $\lambda$ is positive 
and unbounded, approaching infinity quadratically in $\vec{\xi}$.

Proposition 1 A solution $v_{0}(x, y, t)$ of (1.1) is linearly ill-posed in a neighborhood of $\left(x_{0}, y_{0}, t_{0}\right)$ with respect to the exponential perturbation $\exp \left\{i\left(\xi_{1} x+\xi_{2} y\right)\right\}$ if $\vec{\xi}($ or $-\vec{\xi})$ lies in the sector

$$
\arg \left(\nabla v_{0}\right)<\arg (\vec{\xi})<\arg \left(\nabla v_{0}\right)+\alpha
$$

where

$$
\arg \left(\nabla v_{0}\right)=\arctan \left(\frac{\partial_{y} v_{0}\left(x_{0}, y_{0}, t_{0}\right)}{\partial_{x} v_{0}\left(x_{0}, y_{0}, t_{0}\right)}\right)
$$

Note that the upper bound in (2.2) is the argument of the rotated gradient vector, $\arg \left(R \nabla v_{0}\right)=$ $\arg \left(\nabla v_{0}\right)+\alpha$.

Proof: Using the summation convention, we write out (1.1) as

$$
\frac{\partial v}{\partial t}=\frac{1}{|\nabla v|} R_{j k}\left\{\partial_{j} \partial_{k} v-\frac{\partial_{k} v}{|\nabla v|} \frac{\partial_{l} v}{|\nabla v|} \partial_{j} \partial_{l} v\right\} .
$$

Substitute

$$
v=v_{0}(x, y, t)+\epsilon e^{i\left(\xi_{1} x+\xi_{2} y\right)} e^{\lambda t}
$$

into (2.3), equate terms of order $\epsilon$, and freeze coefficients. Then, writing

$$
\lambda(\vec{\xi})=\lambda_{p}(\vec{\xi})+O(|\vec{\xi}|)
$$

to extract the principal part in the growth rate ${ }^{1}$, we calculate that

$$
\lambda_{p}(\vec{\xi})=|\vec{\xi}|^{2} \cos \alpha-\langle R \vec{a}, \vec{\xi}\rangle\langle\vec{a}, \vec{\xi}\rangle=(\vec{a} \times \vec{\xi}) \cdot(R \vec{a} \times \vec{\xi})
$$

where $\langle\cdot, \cdot\rangle$ denotes the Euclidean inner product and $\vec{a}=\nabla v_{0} /\left|\nabla v_{0}\right|$. The second equality in (2.4) is a consequence of Lagrange's vector identity and yields the growth rate in terms of vector dot and cross products. Observe that $\lambda_{p}$ vanishes if $\vec{\xi}$ is parallel to $\vec{a}$ or $R \vec{a}$. A nonzero, homogeneous quadratic form such as (2.4) can vanish along only two directions. Therefore $\lambda_{p}(\xi)$ cannot change sign within the wedge $(2.2)$. We may see that $\lambda_{p}(\xi)$ is positive in this wedge, and negative outside it, by observing that on the circle $\{|\vec{\xi}|=1\}$, the first term in (2.4) is constant while the second term assumes its maximum where the circle intersects the line that bisects the wedge, $\{\arg \vec{\xi}=\arg \vec{a}+\alpha / 2\}$.

As may be seen from the proof, the two angles that bound the wedge (2.2) represent characteristic directions in the $(x, y)$-plane of the steady-state equation

$$
\operatorname{div}\left(R \frac{\nabla v}{|\nabla v|}\right)=0
$$

$\overline{1}$ Note that if $v_{0}(x, y)$ is an equilibrium solution of (1.1), then $\lambda=\lambda_{p}$, i.e. the exponential growth rate equals the principal part. 
consequently, the steady state equation is hyperbolic.

For the special case of $\alpha=0$, the rotation matrix in (1.1) becomes the identity, $R=I$, and (1.1) has a functional that is monotone decreasing (subject to natural boundary conditions),

$$
E=\int|\nabla v| d x d y, \quad \frac{d E}{d t}=-\int\left[\operatorname{div}\left(\frac{\nabla v}{|\nabla v|}\right)\right]^{2} d x d y \leq 0
$$

This special case is related to models for geometric motion by mean curvature [11]. Generalizations of geometric evolution equations have been considered in the field of image processing [2,5,8,20]. These latter models, generally called Perona-Malik equations [24], are based on a different class of generalizations than (1.1); however, we will show that they share some important properties. For (1.1) with $0<\alpha<\pi / 2$ there is no decreasing functional corresponding to (2.6). However, we shall identify a Liapunov function for one-dimensional solutions.

\subsection{The continuous one-dimensional model}

For any nonzero $\vec{a}=\left(a_{1}, a_{2}\right)^{T} \in \mathbf{R}^{2}$, the linear function

$$
v(x, y)=a_{1} x+a_{2} y
$$

is a solution of the steady-state equation (2.5), one which describes uniform shearing. In this paper we study only one-dimensional perturbations of $(2.7)$, i.e. solutions of the form

$$
v(x, y, t)=a_{1} x+a_{2} y+w(x, t) .
$$

Without loss of generality, throughout this paper we take $|\vec{a}|=1$ in (2.8), and for simplicity we take advantage of rotational invariance of the equation (1.1) to make the one-dimensional perturbation be in the $x$-direction. Observe that for functions of this form $\partial_{y} v=a_{2}$; thus, provided that $a_{2} \neq 0, \nabla v$ will never vanish, thereby avoiding the singularity in (1.1). This ansatz is motivated partly by having observed such one-dimensional solutions as the largetime limit of two-dimensional simulations of (1.1) and partly by our desire, in this initial investigation of ill-posed problems, to work in a context where much of the behavior can be derived rigorously.

To examine ill-posedness of the one-dimensional problem, suppose that $w(x, t)$ in $(2.8)$ is an exponential, $w=e^{i \xi_{1} x+\lambda t}$. Since $\nabla v_{0}=\vec{a}$ and $\arg \vec{\xi}=0,(2.2)$ may be simplified to show that one-dimensional perturbations of the steady-state solution (2.7) are ill-posed if

$$
-\alpha<\arg \vec{a}<0
$$

For contrast, if $-\pi<\arg \vec{a}<-\alpha$ or if $0<\arg \vec{a}<\pi-\alpha$, then (2.7) is well-posed in the one-dimensional context. In this respect, the one-dimensional problem differs greatly from 


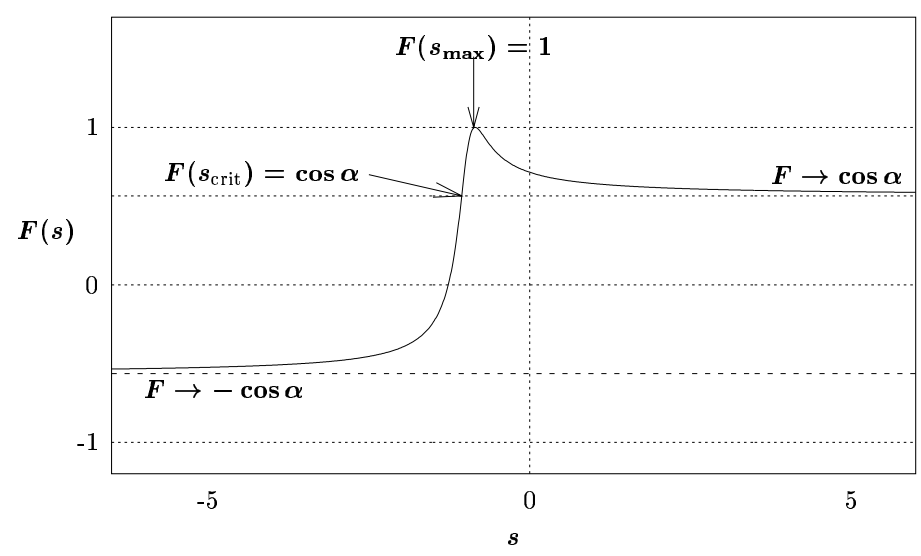

Fig. 2. The nonlinear flux function $F(s)$ for $0<\phi<\alpha$ (Case 1).

the two-dimensional problem: (1.1) is always ill-posed when plane waves in all directions are allowed as perturbations of $(2.7)$.

On substitution of (2.8) into (1.1), we obtain

$$
\frac{\partial w}{\partial t}=\frac{\partial}{\partial x}\left(F\left(w_{x}\right)\right)
$$

where

$$
F(s)=\left\langle R^{T} \overrightarrow{\mathrm{e}}_{1}, \frac{\vec{a}+s \overrightarrow{\mathrm{e}}_{1}}{\left|\vec{a}+s \overrightarrow{\mathrm{e}}_{1}\right|}\right\rangle
$$

here $R^{T}$ is the transpose (and inverse) of $(2.1)$, and $\overrightarrow{\mathrm{e}}_{1}=(1,0)^{T}$. Recalling that $|\vec{a}|=1$, we define an angle $\phi=-\arg \vec{a}$ (note the minus sign), so

$$
\vec{a}=(\cos \phi,-\sin \phi)^{T}
$$

without loss of generality we restrict $\phi$ to the range $0 \leq \phi \leq \pi$. We shall consider (2.10) on the interval $0 \leq x \leq 1$ and impose homogeneous Dirichlet boundary conditions on $w$,

$$
w(0, t)=0, \quad w(1, t)=0 .
$$

In terms of the two-dimensional PDE (1.1), we are seeking a solution on the vertical strip $\Omega=$ $\{(x, y) \in[0,1] \times \mathbf{R}\}$ of the particular form (2.8) satisfying boundary conditions $v(0, y, t)=$ $a_{2} y$, and $v(1, y, t)=a_{1}+a_{2} y$. It is interesting to note that in the cases $\phi=0$ and $\phi=\alpha$, the boundary of $\Omega$ is characteristic for the steady-state problem (2.5). Incidentally, the case of periodic boundary conditions for $(2.10)$, i.e. $w(1+x, t)=w(x, t)$, is also covered by our analysis apart from a possible nonzero mean value for $w(x)$.

The properties of PDE (2.10) depend on the form of the nonlinear flux function $F(s)$. Using 
(2.12), in terms of $\phi,(2.11)$ can be rewritten

$$
F(s)=\frac{\cos (\alpha-\phi)+s \cos \alpha}{\sqrt{1+2 s \cos \phi+s^{2}}} .
$$

Figure 2 shows a graph of $F(s)$ for one choice of $\alpha$ and $\phi$. Note that, for every choice of $\alpha$ and $\phi, F(s)$ is a bounded function for all $s$, taking values in the range $-\cos \alpha<F(s) \leq 1$, with the lower bound approached as $s \rightarrow-\infty$. As $s \rightarrow \infty$,

$$
F(s)=\cos \alpha+\frac{\sin \alpha \sin \phi}{s}+O\left(s^{-2}\right) .
$$

The upper limit, $F(s)=1$, is achieved at $s_{\max }$, the unique maximum of $F$, where the two vectors in the inner product (2.11) are parallel: i.e., $\arg \left(\vec{a}+s_{\max } \vec{e}_{1}\right)=-\alpha$. This critical point is given by

$$
s_{\max }=-\sin (\alpha-\phi) / \sin \alpha .
$$

This point separates the two intervals $\left(-\infty, s_{\max }\right)$ and $\left(s_{\max }, \infty\right)$, where $F(s)$ is monotone increasing and decreasing, respectively.

Differentiating the one-dimensional equation (2.10) with respect to $x$, we obtain a nonlinear diffusion equation for the slope $s(x, t) \equiv w_{x}(x, t)$,

$$
\frac{\partial s}{\partial t}=\frac{\partial^{2}}{\partial x^{2}}(F(s)) \quad \text { or } \quad \frac{\partial s}{\partial t}=\frac{\partial}{\partial x}\left(D(s) \frac{\partial s}{\partial x}\right),
$$

where $D(s) \equiv F^{\prime}(s)$ is the nonlinear diffusion coefficient. For $s<s_{\max }, D(s)>0$ and hence $(2.17)$ is a linearly well-posed nonlinear forward diffusion equation, while for $s>s_{\max }$, $D(s)<0$ and (2.17) is a linearly ill-posed backward diffusion equation (see Figure $3 \mathrm{~b}$ ). We observe that for $F(s)$ given by (2.14), the corresponding diffusion coefficient is

$$
D(s)=-\frac{\sin \alpha \sin \phi}{\left(1+2 s \cos \phi+s^{2}\right)^{3 / 2}}\left(s-s_{\max }\right) .
$$

The one-dimensional Perona-Malik equation [20], $w_{t}=\left(\rho\left(w_{x}^{2}\right) w_{x}\right)_{x}$, is of the form (2.17), with $F\left(w_{x}\right)=\rho\left(w_{x}^{2}\right) w_{x}$. For this equation, $F^{\prime}(s)=\rho\left(s^{2}\right)+2 \rho^{\prime}\left(s^{2}\right) s^{2}$ also becomes negative at large $s$ for appropriate functions $\rho\left(s^{2}\right)$ of interest [20].

Equation (2.10) has the Liapunov function

$$
\mathcal{L}=\int_{0}^{1} V\left(w_{x}\right) d x \quad \text { where } \quad V(s)=\int F(s) d s .
$$

Subject to Dirichlet or other appropriate boundary conditions, the Liapunov function is monotone decreasing, with its evolution given by

$$
\frac{d \mathcal{L}}{d t}=-\int_{0}^{1}\left[\partial_{x} F\left(w_{x}\right)\right]^{2} d x \leq 0 .
$$



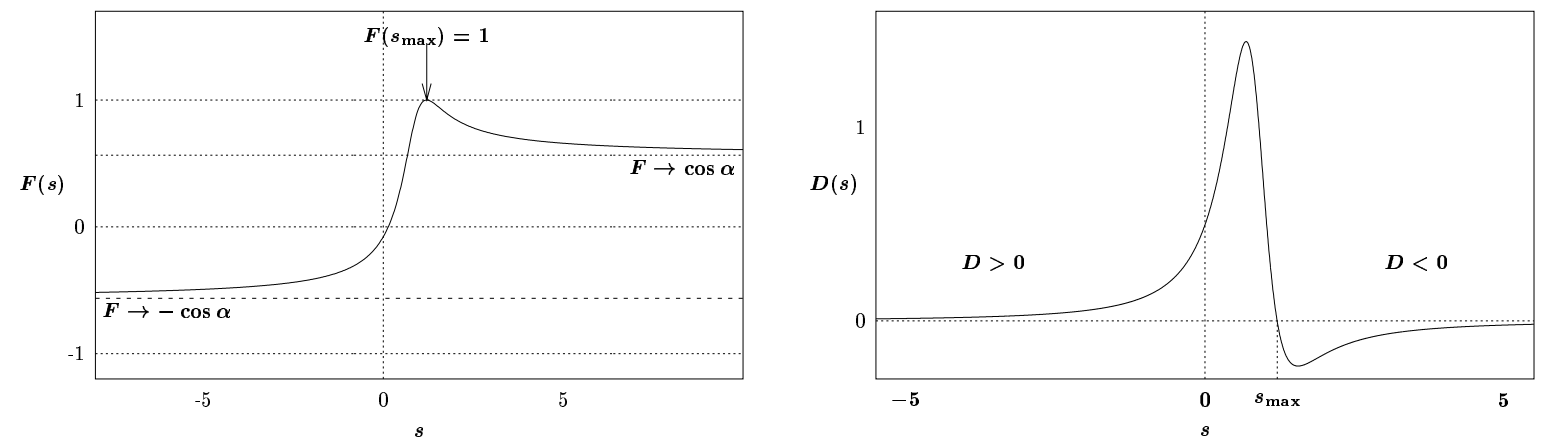

Fig. 3. The nonlinear flux function $F(s)$ and the corresponding nonlinear diffusion coefficient $D(s)=F^{\prime}(s)$ for $\phi>2 \alpha$ (Case 3$)$.

Up to an additive constant, the anti-derivative of $F(s)$ in (2.14) is given by

$$
V(s)=\sin \alpha \sin \phi \ln \left(\cos (\phi)+s+\sqrt{1+2 s \cos \phi+s^{2}}\right)+\cos \alpha \sqrt{1+2 s \cos \phi+s^{2}} .
$$

We define $s_{\text {crit }}$ as the value of the slope in $\left(-\infty, s_{\max }\right)$ where $F(s)$ equals its limit as $s \rightarrow \infty$ : i.e., $F\left(s_{\text {crit }}\right)=\cos \alpha$, or $\arg \left(\vec{a}+s_{\text {crit }} \overrightarrow{\mathrm{e}}_{1}\right)=-2 \alpha$ (see Figure 2 ). This value is given by

$$
s_{\text {crit }}=-\sin (2 \alpha-\phi) / \sin (2 \alpha) \text {. }
$$

We will show that the long-time behavior of the discrete model of (2.10) is related to whether $s_{\max }$ and $s_{\text {crit }}$ are positive or negative. We distinguish three cases as follows, indicating the corresponding relations between $\alpha$ and $\phi$, for each:

$$
\begin{array}{lll}
\text { Case 1: } & s_{\text {crit }}<s_{\max }<0 & 0<\phi<\alpha \\
\text { Case 2 }: & s_{\text {crit }}<0<s_{\max } & \alpha<\phi<2 \alpha \\
\text { Case 3: } & 0<s_{\text {crit }}<s_{\max } & 2 \alpha<\phi<\pi
\end{array}
$$

Note that in Case 1, we have $F^{\prime}(0)<0$ (see Fig. 2), so the trivial equilibrium solution $w \equiv 0$ is ill-posed to one-dimensional perturbations. More generally, we claim that any possible smooth solution must be ill-posed in Case 1. To see this, observe that the boundary conditions (2.13) force solutions to maintain zero average slope, $\int w_{x} d x=0$. Hence in Case 1 , every non-trivial continuous $w(x)$ will be ill-posed in some subset of $0 \leq x \leq 1$, where its slope is positive. This argument for the one-dimensional problem (2.10) independently rederives and generalizes the earlier linear stability result (2.9). In contrast, for Cases 2 and 3 the trivial solution of (2.10) is well-posed. Moreover, for initial data with $\max \left(w_{x}\right)<s_{\max }$, (2.10) is strictly forward parabolic. Consequently (i) a maximum principle for $w_{x}$ can be established, (ii) the $L_{2}$ norm of $w_{x}$ evolves according to

$$
\frac{d}{d t} \int_{0}^{1} w_{x}^{2} d x=-\int_{0}^{1} D\left(w_{x}\right) w_{x x}^{2} d x \leq 0, \quad \text { for } w_{x}<s_{\max }
$$


and (iii) this class of solutions converge to $w=0$ as $t \rightarrow \infty$. While Case 1 can be distinguished from Cases 2 and 3 by the respective ill- or well-posedness of $w=0$, we will show that Cases 2 and 3 differ in whether or not $w=0$ is the unique equilibrium solution of the discretized version of $(2.10)$.

\subsection{The semi-discrete one-dimensional problem}

As discussed in the introduction, we consider a continuous-time/discrete-space, approximation to $(2.10,2.13)$. Specifically, given a positive integer $N \geq 2$, let $\Delta x=1 / N$ be the uniform spacing of grid points in a finite-difference discrete solution $w_{n}(t) \approx w(n \Delta x, t)$, for $n=0,1,2, \ldots, N$. The discrete solution evolves according to the coupled system of $(N-1)$ nonlinear ordinary differential equations for $n=1,2, \ldots N-1$,

$$
\frac{d w_{n}}{d t}=\frac{1}{\Delta x}\left\{F\left(\frac{w_{n+1}-w_{n}}{\Delta x}\right)-F\left(\frac{w_{n}-w_{n-1}}{\Delta x}\right)\right\}
$$

where the boundary conditions (2.13) become

$$
w_{0}=0, \quad w_{N}=0 .
$$

For $N=2,(2.25,2.26)$ reduces to a single first order equation for $w_{1}$; similarly for $N=3$, the general model reduces to an autonomous phase plane system for $w_{1}$ and $w_{2}$. Recently and independently, work on these low-dimensional models was done in [35] for the study of clustering instabilities in granular gases. Our analysis of the solutions of $(2.25,2.26)$ applies for all $N \geq 2$, but our focus will be the consideration of large values of $N$ and the continuum limit, $N \rightarrow \infty$.

For brevity, we will write the arguments of $F(\cdot)$ in $(2.25)$ as

$$
w_{n+1 / 2}^{\prime} \equiv \frac{w_{n+1}-w_{n}}{\Delta x}
$$

This is a second-order-accurate centered finite-difference approximation of the spatial derivative, i.e. $w_{n+1 / 2}^{\prime}(t)=w_{x}\left(\left(n+\frac{1}{2}\right) \Delta x, t\right)+O\left(\Delta x^{2}\right)$. This discrete system has a Liapunov function analogous to (2.19),

$$
\mathcal{L}\left(w_{n}\right)=\sum_{n=0}^{N-1} V\left(w_{n+1 / 2}^{\prime}\right) \Delta x
$$

Using summation by parts, it can be shown similarly that the discrete Liapunov function is monotone decreasing, 


$$
\begin{aligned}
\frac{d}{d t}\left(\sum_{n=0}^{N-1} V\left(w_{n+1 / 2}^{\prime}\right) \Delta x\right) & =\sum_{n=0}^{N-1}\left[F\left(w_{n+1 / 2}^{\prime}\right)\left(\frac{d w_{n+1}}{d t}-\frac{d w_{n}}{d t}\right)\right] \\
& =-\sum_{n=1}^{N-1}\left(\frac{F\left(w_{n+1 / 2}^{\prime}\right)-F\left(w_{n-1 / 2}^{\prime}\right)}{\Delta x}\right)^{2} \Delta x \leq 0 .
\end{aligned}
$$

Formally, the discrete model (2.25) converges to the PDE (2.10) as $\Delta x \rightarrow 0$ up to $O\left(\Delta x^{2}\right)$ errors. Retaining terms through order $O\left(\Delta x^{4}\right)$, we derive the effective PDE for (2.25),

$$
\frac{\partial w}{\partial t}=\frac{\partial}{\partial x}\left\{F\left(w_{x}\right)+\frac{\Delta x^{2}}{24} \frac{1}{w_{x x}} \frac{\partial}{\partial x}\left(w_{x x} \frac{\partial}{\partial x}\left[F\left(w_{x}\right)\right]\right)\right\}+O\left(\Delta x^{4}\right) .
$$

Linearizing this equation about $w \equiv 0$, we obtain

$$
\frac{\partial w}{\partial t}=D(0)\left(\frac{\partial^{2} w}{\partial x^{2}}+\frac{\Delta x^{2}}{12} \frac{\partial^{4} w}{\partial x^{4}}\right)+O\left(\Delta x^{4}\right)
$$

For $D(0)<0$, equation (2.31) contains a destabilizing second-order term and a regularizing fourth-order term. This balance of terms occurs in many models of physical systems, including the Cahn-Hilliard equation for binary mixtures [7,29] and the Kuramoto-Sivashinsky equation [15] from combustion theory, image processing models [20,8,2], biological systems $[23,21]$, and spatially discrete mechanical systems $[28,26]$. Solutions of these equations exhibit regions where the solution is smooth, which are separated by thin layers with large gradients; for large times these layers eventually merge together $[29,30]$. We observe analogous coarsening behavior in solutions of (2.25).

For $D(0)>0$ the fourth order linearized modified PDE (2.31) is high-frequency unstable. However, as noted in the introduction, the discrete finite difference model (2.25) eliminates the dynamics of all length scales smaller than the fundamental grid-spacing. Consequently the spatially discrete system (2.25) is not subject to such unbounded growth rates at high frequencies.

Since $F(s)$ is bounded, solutions of $(2.25)$ exist for all time and grow at most linearly in time. We show that, in fact, independent of $\Delta x=1 / N$, the solution is bounded for all time in the max norm: i.e. there exists a $C$ such that

$$
\max _{n}\left|w_{n}(t)\right| \leq C
$$

In view of the boundary condition $(2.26)$, boundedness of $w_{n}(t)$ is an immediate consequence of the following proposition.

Proposition 2 If $w(t)$ is a solution of (2.25) and if $S_{0}=\min _{n} w_{n+1 / 2}^{\prime}(0)$, then for all $n$ and all $t \geq 0$

$$
w_{n+1 / 2}^{\prime}(t) \geq \min \left(s_{\text {crit }}, S_{0}\right)
$$


Note that if $w(x, t)$ were a twice differentiable solution of (2.10), then $w$ would satisfy the stronger estimate $w_{x}(x, t) \geq \min \left(s_{\max }, \bar{S}_{0}\right)$ where $\bar{S}_{0}=\inf _{x} w_{x}(x, 0)$. Indeed, since $F(s)$ is monotone increasing on $\left(-\infty, s_{\max }\right)$, the maximum principle gives this estimate. However, because of the discretization, solutions of (2.25) do not satisfy this stronger estimate. Nevertheless, the derivation of (2.32) is modeled on the proof of the maximum principle.

Proof: For brevity let $s_{*}$ denote the RHS of (2.32). Obviously, (2.32) is initially satisfied at time $t=0$. Suppose that for all grid points and for all $t \leq t_{*}$ we have $w^{\prime}(t) \geq s_{*}$, and suppose that for some grid point $n$, we have $w_{n+1 / 2}^{\prime}\left(t_{*}\right)=s_{*}$. Then, taking differences of (2.25), we calculate that at this point and $t=t_{*}$,

$$
\left.\frac{d w_{n+1 / 2}^{\prime}}{d t}\right|_{t=t_{*}}=\frac{1}{\Delta x^{2}}\left(F\left(w_{n+3 / 2}^{\prime}\right)-2 F\left(s_{*}\right)+F\left(w_{n-1 / 2}^{\prime}\right)\right) .
$$

We claim that

$$
F\left(s_{*}\right) \leq F\left(w_{n+3 / 2}^{\prime}\right)
$$

and similarly for $F\left(w_{n-1 / 2}^{\prime}\right)$, from which it follows that at $t=t_{*}$,

$$
\left.\frac{d w_{n+1 / 2}^{\prime}}{d t}\right|_{t=t_{*}} \geq 0
$$

To prove the claim, observe that $s_{*} \leq w_{n+3 / 2}^{\prime}$. If $w_{n+3 / 2}^{\prime} \leq s_{\text {crit }}$, then (2.33) follows from the fact that $F(s)$ is monotone on an interval containing $\left(-\infty, s_{\text {crit }}\right)$. On the other hand, if $s_{\text {crit }} \leq w_{n+3 / 2}^{\prime}$, then

$$
F\left(s_{*}\right) \leq F\left(s_{\text {crit }}\right) \leq F\left(w_{n+3 / 2}^{\prime}\right),
$$

the latter inequality in (2.35) being apparent from Figure 2.

By itself, condition (2.34) is not sufficient to exclude the possibility that $w_{n+1 / 2}^{\prime}(t)<s_{*}$ for some $t>t_{*}$. However, as in the proof of the maximum principle [18], we may derive (2.32) from the above argument by taking the limit of slightly modified functions for which (2.34) becomes a strict inequality.

\section{The discrete steady-state solutions}

The trivial solution, $w_{n} \equiv 0$, is an equilibrium of $(2.25)$. In this section we determine the nontrivial equilibrium solutions of (2.25). We show that the number of solutions depends on $\phi$, corresponding to the three cases identified in (2.23). 


\subsection{Equilibrium solutions of the discrete model}

If an $(N+1)$-component vector $\left\{w_{n}\right\}$ is an equilibrium solution of $(2.25,2.26)$ then there is a constant $\bar{F}$ such that the slopes (2.27) all satisfy

$$
F\left(w_{n+1 / 2}^{\prime}\right)=\bar{F}, \quad n=0,1, \ldots, N-1 .
$$

From the boundary conditions (2.26), the $N$ values of the discrete slope, $\left\{w_{n+1 / 2}^{\prime}\right\}$, are also subject to the global constraint

$$
\sum_{n=0}^{N-1} w_{n+1 / 2}^{\prime}=0
$$

This condition is the discrete analogue of $\int w_{x} d x=0$, the consequence of the homogeneous Dirichlet boundary conditions (2.13). Thus, we have identified equilibrium solutions $\left\{w_{n}\right\}$ of $(2.25,2.26)$ with solutions $\left(\left\{w_{n+1 / 2}^{\prime}\right\}, \bar{F}\right)$ of $(3.1,3.2)$.

Considering (3.1) alone, we note that nontrivial solutions are possible only when $F(s)=\bar{F}$ has multiple solutions. If $\cos \alpha<\bar{F}<1$, there are two values of the slope $s$, call them $s_{1}$ and $s_{2}$, such that $F(s)=\bar{F}$,

$$
F\left(s_{1}\right)=F\left(s_{2}\right)=\bar{F} .
$$

From the properties of $F(s)$ given by (2.14) it is clear that $s_{1}$ and $s_{2}$ must bracket $s_{\max }$. When $\bar{F} \approx 1$ both roots are close to $s_{\max }$; as $\bar{F}$ decreases the roots separate continuously. As $\bar{F} \rightarrow \cos \alpha$, one solution of (3.3) becomes large, $s_{1} \rightarrow \infty$, while the other one approaches the limit $s_{2} \rightarrow s_{\text {crit }}$. Using (2.14) we can eliminate $\bar{F}$ in (3.3) to express $s_{1}$ in terms of $s_{2}$,

$$
s_{1}=H\left(s_{2}\right) \equiv \frac{\frac{\sin (2 \alpha-2 \phi)}{\sin (2 \alpha)}-s_{\text {crit }} s_{2}}{s_{\text {crit }}-s_{2}}
$$

This formula shows that, as $\bar{F}$ varies, $\left(s_{1}, s_{2}\right)$ traces out a portion of a hyperbola, as shown in Figure 4. This hyperbola is necessarily invariant under the interchange of $s_{1}$ and $s_{2}$. Its horizontal and vertical asymptotes are $s_{1,2} \rightarrow s_{\text {crit }}$. Changing the values of $\alpha$ and $\phi$ affects the position of this hyperbola in the plane and changes the number of solutions of the discrete problem $(3.1,3.2)$.

To complete the description of the nontrivial equilibrium solutions, we now examine the constraint (3.2). To enumerate the equilibrium solutions, we define $K$ as the number of grid positions where the slope $w_{n+1 / 2}^{\prime}$ is $s_{1}$; the remaining $N-K$ positions will have slope $s_{2}$. Consequently $(3.2)$ reduces to

$$
K s_{1}+(N-K) s_{2}=0 .
$$




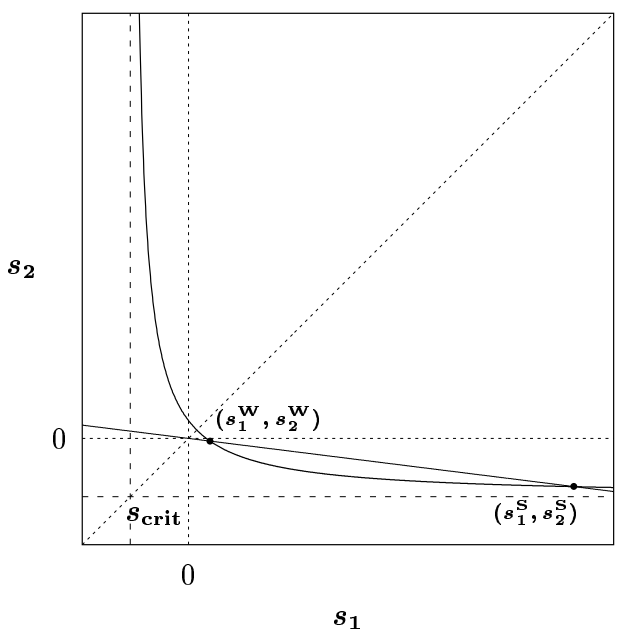

Fig. 4. The construction for the weak $\left(s_{-}^{\mathrm{W}}, s_{+}^{\mathrm{W}}\right)$ and strong $\left(s_{-}^{\mathrm{S}}, s_{+}^{\mathrm{S}}\right)$ solutions in Case 2, given by the intersection points of the hyperbola (3.4) and the line (3.5).
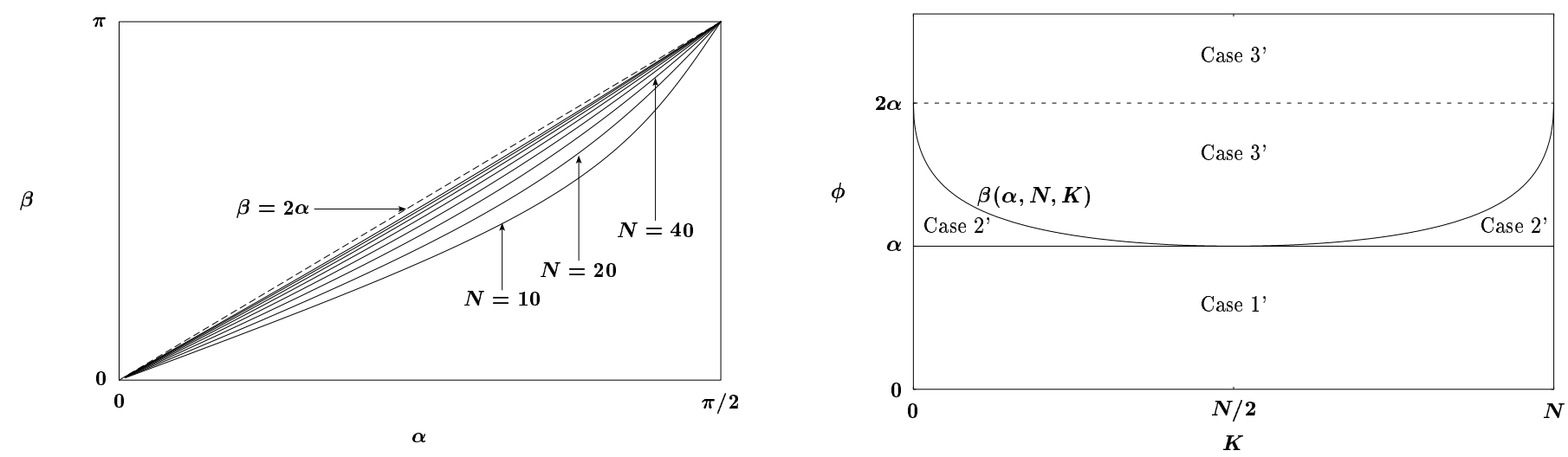

Fig. 5. (left) The upper bound for Case $2^{\prime}, \phi \leq \beta(\alpha, N, 1)$, for $N=10,20,40, \cdots$, and the continuum limit, $\beta \rightarrow 2 \alpha$ as $N \rightarrow \infty$, and (right) $\beta(\alpha, N, K)$ for $K$ in $1 \leq K<N$ with fixed $N$.

Graphically, finding the intersection points of the hyperbola (3.4) and the line (3.5) in the $\left(s_{1}, s_{2}\right)$ plane yields all of the nontrivial equilibrium solutions at given $(\alpha, \phi)$, see Figure 4 . Eliminating $s_{1}$ from $(3.4,3.5)$ yields a quadratic equation for $s_{2}$. This equation has two real solutions if the resulting discriminant is positive,

$$
(N-2 K)^{2} \sin ^{2}(2 \alpha-\phi)+4 K(N-K) \sin (2 \alpha) \sin (2 \alpha-2 \phi) \geq 0 .
$$

The case of equality in (3.6) corresponds to the case when the line (3.5) is tangent to the hyperbola (3.4) and defines an upper bound for $\phi$ in Case 2. We write this bound as the function $\beta, \phi \equiv \beta(\alpha, N, K)$, in terms of $\alpha, N, K$ with $\alpha<\beta<2 \alpha$ (see Figure 5).

Using $\beta(\alpha, N, K)$, we can define three cases for the equilibrium solutions of the discrete problem (2.25) that correspond to the cases given by (2.23). In the limit that $N \rightarrow \infty$, for any fixed $K$, we find that the upper bound for Case 2 is $\phi=\beta \rightarrow 2 \alpha$, corresponding to (2.23) (see Figure 5). For finite $N$, the condition $\phi=\beta(\alpha, N, K)$ defines the degenerate case 


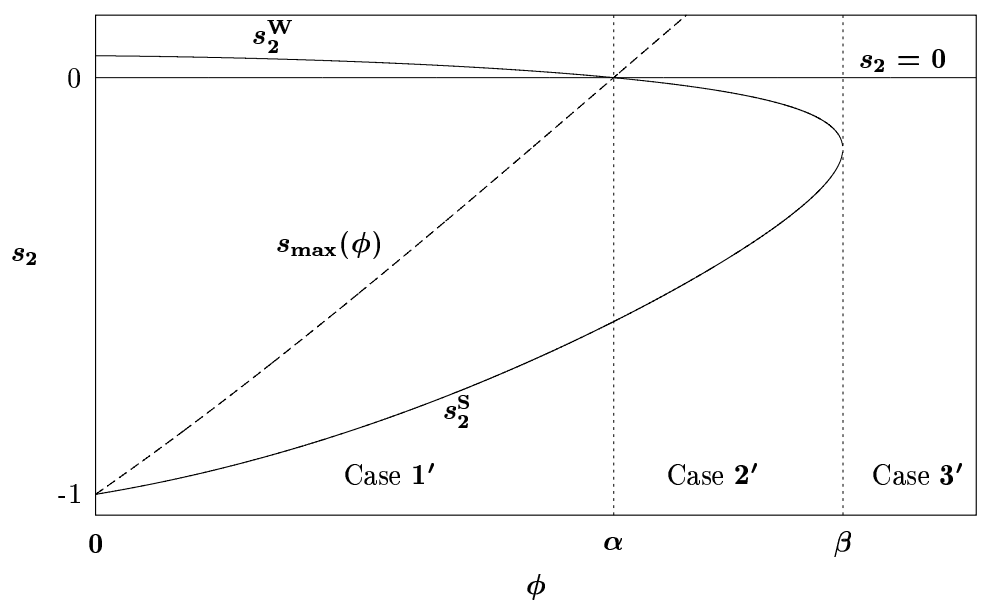

Fig. 6. The bifurcation diagram for the weak, strong, and trivial equilibrium solutions.

where the quadratic for $s_{2}$ has a double root. For every $\phi<\beta$ there are two distinct real roots for $s_{2}$, which we will call the strong and weak solutions (denoted by superscripts $\mathrm{S}$ and $\mathrm{W}), s_{2}^{\mathrm{S}}<s_{2}^{\mathrm{W}}$, see Figure 6 . For the range $0 \leq \phi<\alpha$ corresponding to Case 1 , (see $\left.(2.23)\right), s_{2}^{\mathrm{S}}$ and $s_{2}^{\mathrm{W}}$ have opposite signs, see Figure 6 . At $\phi=\alpha$, the weak solution becomes degenerate, $s_{2}^{\mathrm{W}}=0$, and it intersects the branch of trivial solutions, $w \equiv 0$. As we will describe later, this intersection point is a transverse bifurcation that is connected with a change in stability of these solution branches. For the range, $\alpha<\phi<\beta$, corresponding to Case 2 (as $N \rightarrow \infty$ ) both strong and weak solutions yield negative slopes for $s_{2}$. Finally, for the range $\phi>\beta$, corresponding to Case 3 (as $N \rightarrow \infty$ ), there is only the trivial solution $w \equiv 0$.

We summarize these results on the classification of nontrivial equilibrium solutions in terms of $s_{2}$ as:

For any $N \geq 2$ and for each $K$ with $1 \leq K<N$ :

Case $1^{\prime}: \quad$ Two solutions, $s_{2}^{\mathrm{S}}<0<s_{2}^{\mathrm{W}} \quad 0<\phi<\alpha$

Case $2^{\prime}$ : $\quad$ Two solutions, $s_{2}^{\mathrm{S}}<s_{2}^{\mathrm{W}}<0 \quad \alpha<\phi<\beta(\alpha, N, K)$

Case $3^{\prime}$ : No nontrivial solutions $\quad \beta(\alpha, N, K)<\phi<\pi$.

Case $1^{\prime}$ is the same as Case 1 of (2.23); Cases $2^{\prime}$ and $3^{\prime}$ approach Cases 2 and 3 of (2.23) as $N \rightarrow \infty$, for which $\beta \rightarrow 2 \alpha$. The corresponding $s_{1}$ values are given by $(3.5), s_{1}=$ $-(N-K) s_{2} / K$. For given values of $N$ and $K$, there are $\left(\begin{array}{l}N \\ K\end{array}\right)$ distinct equilibrium solutions $\left\{w_{n}\right\}$ resulting from different spatial arrangements of the two slopes. Clearly $s_{1}$ and $s_{2}$ must have opposite signs, and if $K \ll N$ then $\left|s_{1}\right| \gg\left|s_{2}\right|$, for example see Figure 7 . These solutions with $K \ll N$ can be described as having jump discontinuities at the grid points with the larger slope $s_{1}$, with the magnitude of the jump being $s_{1} \Delta x=s_{1} / N$; the remainder of the solution is piecewise linear with slope $s_{2}$. We will refer to $s_{1}$ as the "jump" or "shock" slope, and to $s_{2}$ as the "background" or "mean-field" slope, respectively.

At this point we note that the above description actually provides a redundant enumeration of the set of equilibrium solutions. This is due to double counting of the solutions caused by 

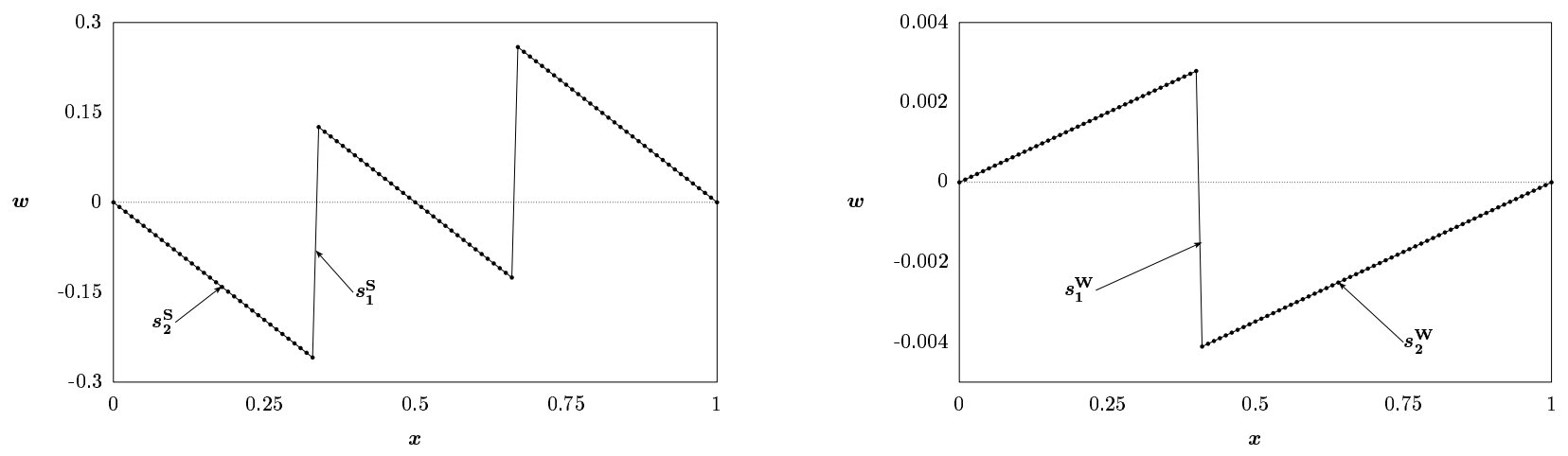

Fig. 7. Two equilibria for Case $1^{\prime}$ with $\alpha=\pi / 8, \phi=\alpha / 2$ and $N=100$; (a) a strong shock solution with $K=2$ jumps, and (b) a much smaller-amplitude weak shock solution for $K=1$.

the symmetry under the interchange,

$$
s_{1} \leftrightarrow s_{2} \quad \text { and } \quad K \leftrightarrow N-K
$$

i.e. if $\left\{s_{1}, s_{2}, K, N-k\right\}$ describes a solution of (3.3) and (3.5), then so does $\left\{s_{2}, s_{1}, N-K, K\right\}$. To eliminate this redundancy, let us define the two slopes in a nontrivial equilibrium solution with one slope, $s_{+}$, that is greater than $s_{\max }, s_{+}>s_{\max }$, and the other less than $s_{\max }$, $s_{-}<s_{\max }$. For enumeration of the solutions, we can associate $s_{+}$with $s_{1}$ and henceforth define $K$ as the number of grid cells with slope $s_{+}$,

$$
K s_{+}+(N-K) s_{-}=0
$$

This description of the two equilibrium slopes will be of particular importance for the later analysis of the stability and dynamics of solutions of (2.25). This is because $s_{+}>s_{\max }$ corresponds to the ill-posed regime for the PDE (2.17) with $D(s)<0$, while $s_{-}<s_{\max }$ corresponds to well-posed behavior with $D(s)>0$. Except in the special case of the Case $1^{\prime}$ weak solution, the descriptions of solutions based on the ill-posed and well-posed slopes, $\left(s_{+}, s_{-}\right)$, are equivalent to the descriptions in terms of the jump and background slopes, $\left(s_{1}, s_{2}\right)$.

We now derive asymptotic estimates for the two families of weak and strong solutions with a single jump, $K=1$, in the limit of large $N$. In this limit, $s_{+}$tends to infinity, and from Figure 2 we see that $s_{-}$tends to $s_{\text {crit }}$; more precisely, by condition (3.5) we obtain

$$
\begin{aligned}
& s_{-}^{\mathrm{S}}=s_{\text {crit }}+O\left(N^{-1}\right)<0, \\
& s_{+}^{\mathrm{S}}=-(N-1) s_{\text {crit }}+O(1)>0 .
\end{aligned}
$$

We will call these solutions with slopes $\left(s_{-}^{\mathrm{S}}, s_{+}^{\mathrm{S}}\right)$ strong shocks. They correspond to the lower branch of solutions shown in the bifurcation diagram Figure 6 , where we note that $\phi=0$ yields $s_{\text {crit }}=-1$, i.e. the endpoint of that branch of the bifurcation diagram.

For $K=1$ as $N \rightarrow \infty$, the other solution is given by 


$$
\begin{aligned}
& s_{-}^{\mathrm{W}}=-s_{0} /(N-1)+O\left(N^{-2}\right), \\
& s_{+}^{\mathrm{W}}=s_{0}+O\left(N^{-1}\right)
\end{aligned}
$$

where $s_{0}$ is the nonzero slope such that $F\left(s_{0}\right)=F(0)$, specifically,

$$
s_{0}=-\sin (2 \alpha-2 \phi) / \sin (2 \alpha-\phi)
$$

We will call these solutions weak shocks. As $N \rightarrow \infty$, these $\left\{w_{n}\right\}$ solutions are small in amplitude, they scale as $O\left(N^{-1}\right) \rightarrow 0$ everywhere, with the size of the jump being $s_{+}^{\mathrm{W}} \Delta x \sim$ $s_{0} / N$ (see Figure 7 ). The weak shocks correspond to the upper branch of solutions in Figure 6. At $\phi=\alpha, s_{0}=0$, and as noted earlier, the weak shock solution coincides with the trivial solution $w_{n} \equiv 0$.

Note that similar results for multiple-jump weak and strong shock solutions can be derived for any fixed $K=1,2,3, \ldots$ in the limit that $N \rightarrow \infty$. Then, given the values of the two equilibrium slopes, $s_{+}$and $s_{-}$, and their spatial distribution, say the values $n_{k}$, with $k=$ $1,2, \ldots, K$, of the grid points where the slopes $s_{+}=w_{n_{k}+1 / 2}^{\prime}$ occur, then the solution $\left\{w_{n}\right\}$ can be reconstructed explicitly from

$$
w_{n}=\sum_{j=0}^{n} w_{j+1 / 2}^{\prime} \Delta x, \quad n=0,1,2, \ldots, N .
$$

\subsection{Comparison of discrete equilibria with generalized solutions of the PDE (2.10)}

As described above, the equilibrium solutions of the discrete problem (2.25) are piecewise linear functions with $K$ finite jumps. For the case of $K=1$ jump, applying the boundary conditions (2.26) and summing the difference quotient $w_{n+1 / 2},(2.27)$, over $n$ yields the equilibrium solution

$$
w_{n}=s_{-}\left[n \Delta x-H\left(n-\left[n_{*}+\frac{1}{2}\right]\right)\right]
$$

where $\Delta x=1 / N, H(\cdot)$ is the Heaviside function, and $n_{*}$ is the value of $n$ for which $w_{n+1 / 2}^{\prime}=$ $s_{+}$. For $N \rightarrow \infty$, this solution is the discrete analogue of a weak solution of the PDE (2.10).

Formally, an equilibrium solution of (2.10) has $\partial_{x} F\left(w_{x}\right)=0$, or equivalently $F\left(w_{x}\right)=\bar{F}$. If $\bar{F}=\cos \alpha$ then there is a family of piecewise linear weak equilibrium solutions with a single finite-jump discontinuity. The mean-field equilibrium slope is the finite solution of $F\left(w_{x}\right)=\cos \alpha$, that is $w_{x}=s_{\text {crit }},(2.22)$. Consequently we can write the equation for an equilibrium solution with a single discontinuity as $w_{x}=s_{\text {crit }}\left[1-\delta\left(x-x_{*}\right)\right]$, where $\delta$ is the Dirac delta function and $0<x_{*}<1$ is the shock position. Integrating this and applying (2.13) yields the steady-state solution

$$
w(x)=s_{\text {crit }}\left[x-H\left(x-x_{*}\right)\right]
$$


This one-parameter family of solutions, parametrized by the shock position in the domain $0<x_{*}<1$, is the continuum limit of (3.14), since as $N \rightarrow \infty, s_{+} \rightarrow \infty$ and $s_{-} \rightarrow s_{\text {crit }}$.

However, (among many other solutions) piecewise linear weak equilibrium solutions can be constructed with any countable number of positive jump discontinuities. We are content to mention in passing the formal similarity between solutions of the discrete system and generalized solutions of the ill-posed PDE (2.10). More definitive statements about the solutions of the PDE require careful analysis $[14,20]$. In the following sections, we study in detail the stability, local instabilities and global dynamics of the discretized model (2.25).

\section{Stability of the equilibria}

The following result on the stability and long time evolution of solutions of (2.25) for the cases defined in (3.7) will be proved in this section.

Proposition 3 On the stability of equilibrium solutions of (2.25):

Case 1' Only strong shock solutions of (2.25) with a single jump discontinuity are stable.

Case $2^{\prime}$ Strong shock solutions with a single discontinuity and the trivial zero solution are the only stable equilibria.

Case $3^{\prime}$ The zero solution is stable and in fact globally attracting.

In Cases $1^{\prime}$ and $2^{\prime}$ for almost every initial condition (i.e., not on the stable manifolds of the unstable equilibria), every solution approaches a stable equilibrium as $t \rightarrow \infty$.

\subsection{The Liapunov function: a necessary condition for stability}

We now make use of the Liapunov function (2.28)

$$
\mathcal{L}(w)=\sum_{n=0}^{N-1} V\left(w_{n+1 / 2}^{\prime}\right) \Delta x,
$$

to establish some fundamental stability results for the discrete system (2.25). Recall from (2.29) that $\mathcal{L}$ is monotone decreasing as $w$ evolves. Indeed, $\mathcal{L}$ defines $(2.25)$ as a gradient system in the form

$$
\frac{d w_{n}}{d t}=-\frac{1}{\Delta x} \frac{\partial \mathcal{L}}{\partial w_{n}}, \quad n=1,2, \cdots N-1
$$

In particular, $w$ is an equilibrium solution of $(2.25)$ if and only if it is a critical point of $\mathcal{L}$. $\mathcal{L}$ is bounded from below and tends to infinity as $|w| \rightarrow \infty$. Therefore $\mathcal{L}$ must always have at least one local minimum corresponding to a linearly stable solution. 
In Case $3^{\prime}, w \equiv 0$ is the only critical point of $\mathcal{L}$; thus from the properties of $\mathcal{L}, w \equiv 0$ must be a minimum of $\mathcal{L}$. Therefore, in Case $3^{\prime}$, the trivial solution is stable and globally attracting.

Turning to Cases $1^{\prime}$ and $2^{\prime}$, the following result eliminates most candidates in the search for stable equilibria.

Proposition 4 If $w^{0} \in \mathbf{R}^{N+1}$ is an equilibrium solution of (2.25) derived from (3.3, 3.5) with $K \geq 2$, then $w^{0}$ is not a local minimum of $\mathcal{L}$.

Proof: Consider an equilibrium solution $w^{0}$ with $s_{+}, s_{-}$given by $(3.4,3.5)$ for a given $K \geq 2$. To demonstrate that such a solution is not stabl, we show that $\mathcal{L}$ is not a minimum at $w^{0}$ by differentiating along an appropriate curve of vectors in $\mathbf{R}^{N+1},\{w(q)\}$, through $w^{0}$. We construct this one-parameter family of near-equilibrium states by perturbing two of the $K$ values where the slope is $w_{n+1 / 2}^{\prime}=s_{+}$(at points $n=n_{1}$ and $\left.n=n_{2}\right)$ then the finite difference quotients $w_{n+1 / 2}^{\prime}(q)$ satisfy

$$
w_{n+1 / 2}^{\prime}(q)=\left\{\begin{array}{cl}
s_{+}+q & \text { if } n=n_{1}, \\
s_{+}-q & \text { if } n=n_{2}, \\
s_{+} & \text {if } n=n_{k} \text { for } k=3,4, \ldots K \\
s_{-} & \text {otherwise, }
\end{array}\right.
$$

where we note that for $q=0$, we recover the equilibrium, $w(0)=w^{0}$, while the constraint (3.2) is satisfied for all $q$. Then the Liapunov function is

$$
\mathcal{L}(w(q))=\frac{1}{N}\left[V\left(s_{+}+q\right)+V\left(s_{+}-q\right)+(K-2) V\left(s_{+}\right)+(N-K) V\left(s_{-}\right)\right] .
$$

At $q=0$ the first derivative of $\mathcal{L}(w(q))$ vanishes, and the second derivative satisfies

$$
\left.\frac{d^{2} \mathcal{L}}{d q^{2}}\right|_{q=0}=\frac{2}{N} V^{\prime \prime}\left(s_{+}\right)=\frac{2}{N} F^{\prime}\left(s_{+}\right)<0,
$$

where the final inequality follows from $s_{+}>s_{\max }$. Consequently we conclude that (4.2) with $q=0$ is not a local minimum of $\mathcal{L}$ and hence $w^{0}$ with $K \geq 2$ is an unstable equilibrium.

\subsection{Linear stability analysis}

Having used the Liapunov function to establish the instability of all equilibria with more than one jump, we turn to linear stability to analyze the trivial solution and single jump ( $K=1$ ) equilibria. 
First, at the trivial solution $w_{n} \equiv 0$, the linearization of $(2.25)$ is

$$
\frac{d w}{d t}=\mathbf{L} w \equiv F^{\prime}(0) \frac{w_{n+1}-2 w_{n}+w_{n-1}}{\Delta x^{2}}
$$

where $\mathbf{L} \equiv N^{2} F^{\prime}(0) \mathbf{T}$ is the $(N-1) \times(N-1)$ symmetric tridiagonal matrix with

$$
\mathbf{T}=\left(\begin{array}{rrrrrr}
-2 & 1 & & & & \\
1-2 & 1 & & & \\
& 1 & -2 & 1 & & \\
& & \ddots & \ddots & \ddots & \\
& & & 1 & -2 & 1 \\
& & & & 1 & -2
\end{array}\right)
$$

The matrix $\mathbf{T}$ is the standard finite difference centered second derivative operator with homogeneous Dirichlet boundary conditions [16]. Since $\mathbf{T}$ is negative definite, with eigenvalues $\sigma_{j}=-4 \sin ^{2}\left(\frac{1}{2} \pi j / N\right)$ for $j=1,2, . . N-1$, the trivial solution of $(2.25)$ is stable if $F^{\prime}(0)>0$ - in Cases $2^{\prime}$ and $3^{\prime}$ - and is unstable if $F^{\prime}(0)<0$ - in Case $1^{\prime}$.

To complete the stability analysis, we examine the linearization of (2.25) at single jump $(K=1)$ weak and strong shock equilibria for Cases $1^{\prime}, 2^{\prime}$. We will summarize the results below in Proposition 6 . Note that from Proposition 4, we can already eliminate the possibility that the single-jump weak shock solution $\left(s_{+}^{\mathrm{W}}, s_{-}^{\mathrm{W}}\right)$, is a stable equilibrium in Case $1^{\prime}$, since from (3.8) it maps onto a solution with $s_{+}>s_{\max }$ with $K=N-1>2$, however we will mention it in the discussion for completeness.

The linearization of (2.25) may be analyzed in terms of matrices containing two blocks similar-in-form to (4.5). To facilitate the calculation, we introduce the notation $\mathbf{T}_{N-1}^{(d d)}$ for the matrix (4.5); the subscript of course specifies the dimension, and the superscripts refer to the Dirichlet boundary conditions at both end points of the interval. Extending this notation, we shall write $\mathbf{T}_{M}^{(d n)}$ for the analogous $M$-dimensional operator with Dirichlet boundary conditions at the left endpoint and Neumann boundary conditions at the right endpoint: i.e., the $M \times M$ matrix with rows as in (4.5) except with the last row replaced by

$$
\left(\begin{array}{lllll}
0 & \ldots & 0 & 1 & -1
\end{array}\right)
$$

Similarly, $\mathbf{T}_{M}^{(n d)}$ is obtained by modifying the first row of $\mathbf{T}_{M}^{(d d)}$, and $\mathbf{T}_{M}^{(n n)}$ by modifying both the first and last rows.

For an equilibrium with one jump located at grid point $n_{1}=I$, that is $w_{I+1 / 2}^{\prime}=s_{+}$, the linearization of (2.25) may be viewed as a perturbation of a block diagonal matrix,

$$
\mathbf{T}_{0}=\operatorname{diag}\left(\mathbf{T}_{I}^{(d n)}, \mathbf{T}_{N-I-1}^{(n d)}\right)
$$


Specifically, the linearization is given by the symmetric tridiagonal operator

$$
\mathbf{L}(\epsilon)=N^{2} F^{\prime}\left(s_{-}\right)\left(\mathbf{T}_{0}-\epsilon \mathbf{P}\right)
$$

where

$$
\epsilon=-\frac{F^{\prime}\left(s_{+}\right)}{F^{\prime}\left(s_{-}\right)},
$$

and the perturbation $\mathbf{P}$ is given by

$$
\mathbf{P}=\operatorname{diag}\left(\mathbf{0}_{I-1}, \mathbf{A}, \mathbf{0}_{N-I-2}\right),
$$

with $\mathbf{0}_{M}$ denoting the $M \times M$ zero matrix and $\mathbf{A}$ the $2 \times 2$ matrix

$$
\mathbf{A}=\left(\begin{array}{rr}
-1 & 1 \\
1 & -1
\end{array}\right)
$$

Note that $\mathbf{P}$ has three blocks, the $2 \times 2$ middle block overlapping the corners of the two blocks in (4.7). Here we have assumed that $2 \leq I \leq N-3$; the cases with a jump adjacent to either endpoint, which are simpler, are left for the reader.

Proposition $5 \mathbf{T}_{0}-\epsilon \mathbf{P}$ is negative definite if $-\infty<\epsilon<(N-1)^{-1}$ and has one positive eigenvalue if $\epsilon>(N-1)^{-1}$.

Proof: We shall show that

$$
\operatorname{det}\left[-\left(\mathbf{T}_{0}-\epsilon \mathbf{P}\right)\right]=1-(N-1) \epsilon
$$

As shown by Givens (see [16]), the determinant $d_{N-1}$ of an $(N-1) \times(N-1)$ symmetric, tridiagonal matrix with entries $\mathbf{M}=\left\{m_{i j}\right\}$ is generated recursively by $d_{j}=m_{j j} d_{j-1}-$ $\left(m_{j-1, j}\right)^{2} d_{j-2}$ for $j=2,3, \ldots, N-1$ with $d_{0}=1$ and $d_{1}=m_{11}$. In applying this algorithm to $\mathbf{M}=-\left(\mathbf{T}_{0}-\epsilon \mathbf{P}\right)$, for $j=2, \ldots, I-1$ and for $j=I+2, \ldots, N-1$ the recursion relation is

$$
d_{j}=2 d_{j-1}-d_{j-2}
$$

while for the two values of $j$ between these two ranges

$$
d_{I}=(1-\epsilon) d_{I-1}-d_{I-2} \quad \text { and } \quad d_{I+1}=(1-\epsilon) d_{I}-\epsilon^{2} d_{I-1}
$$

In the first range of $j$, from the recursion relation, we obtain $d_{j}=1+j>0$ for $j=$ $2,3, \cdots, I-1$. In the second range of $j$, after applying the special cases for $j=I$ and $j=I+1$ given above, we find $d_{j}=1-\epsilon j$ for $j=I, \cdots, N-1$. Thus, the determinant is given by $d_{N-1}=1-(N-1) \epsilon$, as claimed.

Further, from Givens' theorem [16], the number of positive eigenvalues of $\mathbf{T}_{0}-\epsilon \mathbf{P}$ is given by the number of sign changes in the sequence $\left\{d_{j}\right\}$. Note that $\mathbf{T}_{0}$ is negative definite, hence 
for $\epsilon=0$ there are no positive eigenvalues. Since $\left\{d_{j}\right\}$ for $j \geq I$ is monotone decreasing, only a single sign change can occur, if $d_{N-1}<0$. Since $d_{N-1}(\epsilon)=0$ has a simple zero for $\epsilon=1 /(N-1)$, the matrix $\mathbf{T}_{0}-\epsilon \mathbf{P}$ has a single positive eigenvalue for $\epsilon>1 /(N-1)$.

Proposition 6 The linear stability of the single jump $(K=1)$ equilibria breaks down into cases as given by (3.7):

Case $1^{\prime}$ : The strong shock solution is stable, while the weak shock is highly unstable with $N-1$ positive eigenvalues.

Case 2': The strong shock is stable, while the weak shock solution is unstable with one positive eigenvalue.

Proof: We begin with the strong shock, $\left(s_{-}^{\mathrm{S}}, s_{+}^{\mathrm{S}}\right)$. It can be shown that $s_{-}^{\mathrm{S}}<s_{\max }$ for $0<\phi<\beta(\alpha, N, 1)$ and for any $N$. Therefore the factor $F^{\prime}\left(s_{-}\right)$in (4.8) is always positive for strong shocks. To estimate $\epsilon=\epsilon_{1}^{\mathrm{S}}$ in (4.9), we use the asymptotic form (3.10) for $\left(s_{-}^{\mathrm{S}}, s_{+}^{\mathrm{S}}\right)$ in the formula (2.18) for $D(s)=F^{\prime}(s)$ :

$$
\epsilon=\epsilon_{1}^{\mathrm{S}} \sim \frac{\left(1+2 s_{\mathrm{crit}} \cos \phi+s_{\mathrm{crit}}^{2}\right)^{3 / 2}}{N^{2}\left(s_{\max }-s_{\text {crit }}\right) s_{\text {crit }}^{2}}=O\left(N^{-2}\right)>0
$$

Consequently since $\epsilon=O\left(N^{-2}\right) \ll(N-1)^{-1}$ as $N \rightarrow \infty$, by Proposition 5, all of the eigenvalues of $\mathbf{L}(\epsilon)$ are negative and the strong shock is stable for Cases $1^{\prime}$ and $2^{\prime}$.

In contrast, for the weak shock $\left(s_{-}^{\mathrm{W}}, s_{+}^{\mathrm{W}}\right)$, in the limit $N \rightarrow \infty$, we find from (2.18) and (3.11) that

$$
\epsilon=\epsilon_{1}^{\mathrm{W}} \sim \frac{s_{0}-s_{\max }}{s_{\max }\left(1+2 s_{\text {crit }} \cos \phi+s_{\text {crit }}^{2}\right)^{3 / 2}}=O(1)>0 .
$$

Therefore, for the weak shock, $\epsilon=O(1) \gg(N-1)^{-1}$ as $N \rightarrow \infty$, and $\mathbf{T}_{0}-\epsilon \mathbf{P}$ is not negative definite but has one positive eigenvalue for both Cases $1^{\prime}$ and $2^{\prime}$. For Case $2^{\prime}, s_{-}^{\mathrm{W}}<s_{\max }$, so $F^{\prime}\left(s_{-}^{\mathrm{W}}\right)$ is positive and the weak shock is unstable with one positive eigenvalues. For Case $1^{\prime}$, $s_{-}^{\mathrm{W}}>s_{\max }$, so the multiplicative factor $F^{\prime}\left(s_{-}^{\mathrm{W}}\right)$ is negative and the $N-1$ negative eigenvalues of $\mathbf{T}_{0}-\epsilon \mathbf{P}$ become $N-1$ unstable positive eigenvalues for $\mathbf{L}(\epsilon)$ for the weak shock.

As was shown above in Proposition 6 , for Case $1^{\prime}$, since it is stable, the strong shock must correspond to a minimum of the Liapunov function $\mathcal{L}$. From $(2.28)$, this value of $\mathcal{L}$ is independent of the position of the jump within the domain. Therefore all $N$ of the single-jump strong shock solutions are stable. The same argument can be applied for the strong shock in Case 2 . These results are independent of the linearized analysis done in this section. Linear stability analysis shows that the position of the shock and the influence of the boundary conditions do weakly effect the values of the eigenvalues, but they do not change the stability of the solutions. 


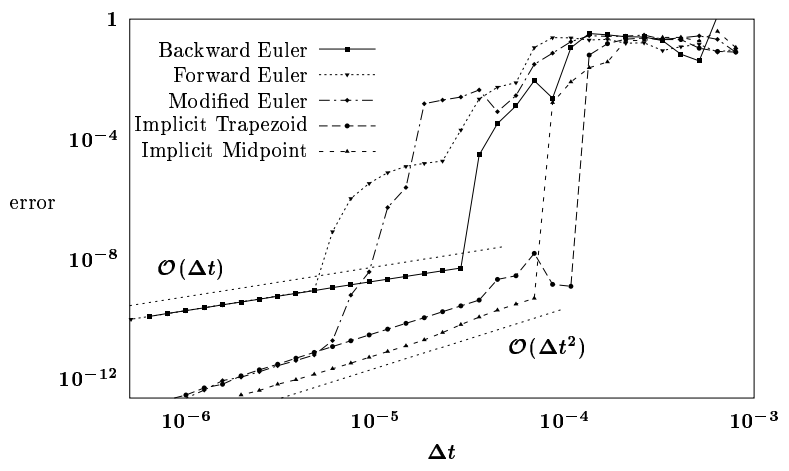

Fig. 8. Convergence of different numerical methods for an initial value problem in Case $1^{\prime}$ for system (2.25).

\section{Representative dynamic simulations}

In this section we present the results of a representative set of numerical simulations of the dynamics of system (2.25). These simulations illustrate some of the differences in behavior in the three cases in Proposition 3. The simulations also guide the analysis of the nonlinear dynamics in the following sections.

We begin with a brief discussion of the numerical methods used for the simulations. As was discussed above, while the continuum PDE (2.10) is ill-posed, for any finite $N$ the discrete system (2.25) is well posed, with global solutions. Subject to typical analytic constraints [16,17], (2.25) can be solved numerically using any appropriate method for integrating systems of coupled ODEs. Figure 8 shows the results of a convergence study as the discrete time-step approaches zero, $\Delta t \rightarrow 0$, for a typical initial value problem. We tested several standard explicit and implicit schemes. For sufficiently small $\Delta t$ all of the methods showed convergence with the expected order of accuracy (see Figure 8). The implicit midpoint method, written in general form as

$$
\frac{w_{n}^{m+1}-w_{n}^{m}}{\Delta t}=\mathcal{F}_{n}\left(\frac{1}{2}\left(w^{m+1}+w^{m}\right)\right)
$$

where $w_{n}^{m} \approx w_{n}\left(t^{m}\right)$ and $t^{m}=m \Delta t$, had the smallest error coefficient and was used for all of the following numerical simulations.

In Figure 9 we consider the evolution of the solution for an initial value problem in Case $1^{\prime}$, with $\alpha=\pi / 8, \phi=\alpha / 2$, and $w_{n}(0)=\sin (\pi n / N)$ for $n=0,1,2, \cdots, N$ with $N=100$. As described above, in Case $1^{\prime}$, the stable steady state is piecewise linear with a single jump. The intermediate dynamics leading up to this state are rather complicated. Figure 9a shows the initial unstable behavior; the solution rapidly develops a large number of jump discontinuities, forming what is sometimes called a "staircase pattern." This stage of the evolution can be compared to spinodal decomposition of binary mixtures [7], where large numbers of phase interfaces develop from an unstable initial state. Figure $9 \mathrm{~b}-\mathrm{d}$ shows the generic mode of evolution for longer times; the sizes of the jump discontinuities evolve on 
(a)

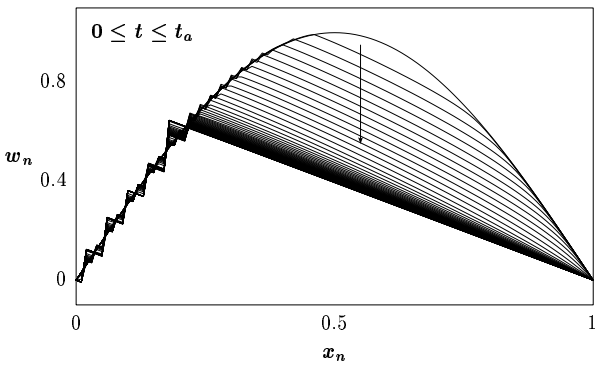

(c)

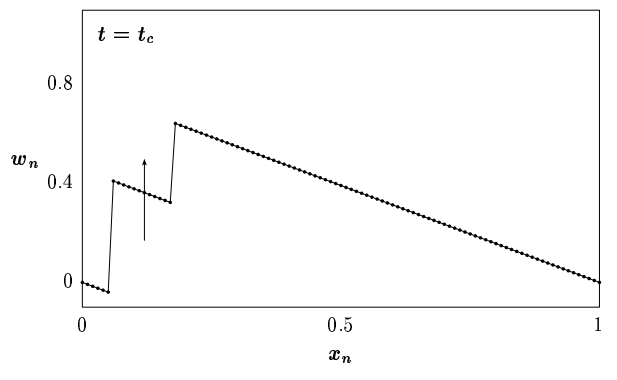

(b)

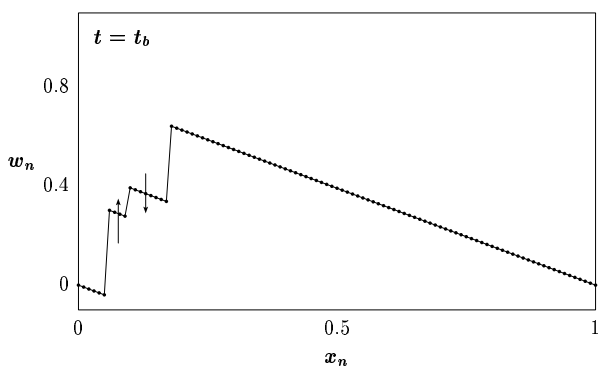

(d)

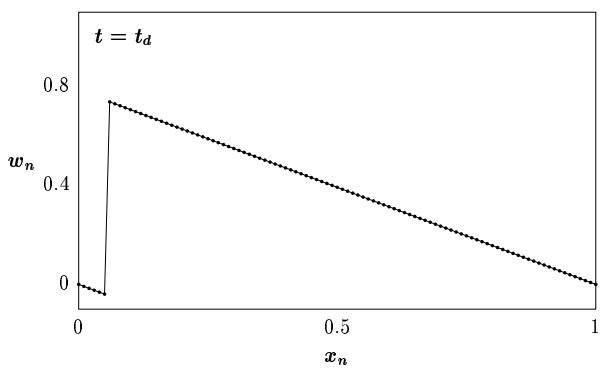

Fig. 9. Evolution for a Case $1^{\prime}$ initial value problem; (a) short time evolution up to time $t_{a}$ captures the unstable phase, where smooth initial data forms lots of discontinuities, $(b-d)$ coarsening behavior at times $t_{b}, t_{c}$ leading to the final steady-state with a single shock, see $t_{d}$.

slower time scales. This regime will be described as coarsening dynamics, where most of the phase interfaces collapse leaving larger intervals where the mean field holds.

Due to the global constraint (3.2), while some of the jumps grow, others must decay. This behavior is illustrated in a different form in Figure 10b, where the values for all of the slopes, $w_{n+1 / 2}^{\prime}(t), n=0 \ldots N-1$ are plotted as functions of time. From this figure we note that at any time $t \gg 0.1$ there are a small number of points with large positive slopes (jumps with $s_{+}>s_{\max }$ ), while most of the grid points have a small negative slope (the mean field with $\left.s_{-}<s_{\max }\right)$. From Figure 10a we observe that the dynamics for (2.25) has a monotone decreasing Liapunov function (2.28) that experiences a sequence of rapid declines coinciding with the collapse of each successive jump. Ultimately, the single-jump, stable, strong shock is approached for $t_{d}<t \rightarrow \infty$ (see Figure 9d). Incidentally, the location of the final jump depends very sensitively on the initial data and on the simulation parameters. In a series of numerical experiments like that of Figure 9, but with perturbed initial data $w_{n}(0)=(1+\epsilon) \sin (\pi n / N)$ with $\epsilon=O\left(10^{-12}\right)$, we found that even such tiny perturbations lead to discontinuous changes in the position of the steady-state jump. This extreme sensitivity is a clear reflection of the ill-posed nature of the underlying problem.

For contrast with Figure 9, we present the evolution of the problem in Case $3^{\prime}$, with $\phi=2.1 \alpha$, starting from the same initial conditions, see Figure 11. Figure 11a shows that for long times, the solution converges to the stable trivial solution, $w=0$. However, since the initial data is partially ill-posed, with the slopes of the initial condition satisfying $w_{n+1 / 2}^{\prime}>s_{\max }$ for some range in $x$, a staircase pattern composed of finite jumps with large slopes develops for $t \approx$ 0.1. For comparison with Figure 10b, Figure 11b shows the more complicated intermediate dynamics for the large slopes in Case $3^{\prime}$, before they all decay to zero. 

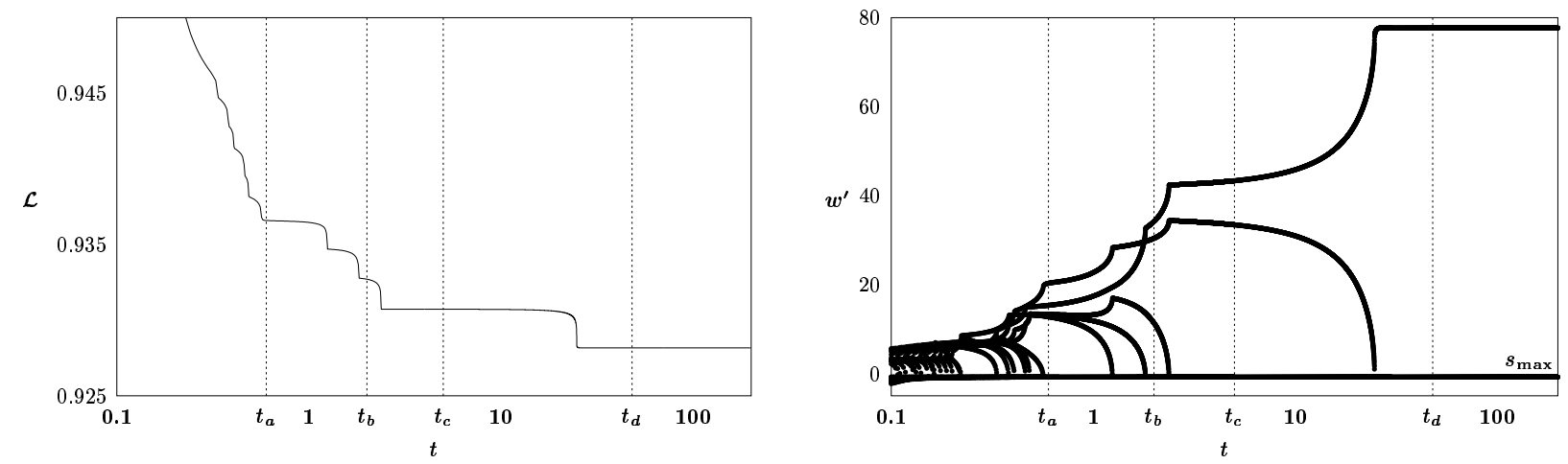

Fig. 10. (a) The evolution of (a) the Liapunov function, and (b) the values of the local slopes $w_{n+1 / 2}^{\prime}$ for $n=0,1,2, \ldots N-1$ plotted as a function of time for the Case $1^{\prime}$ problem in Fig 9.
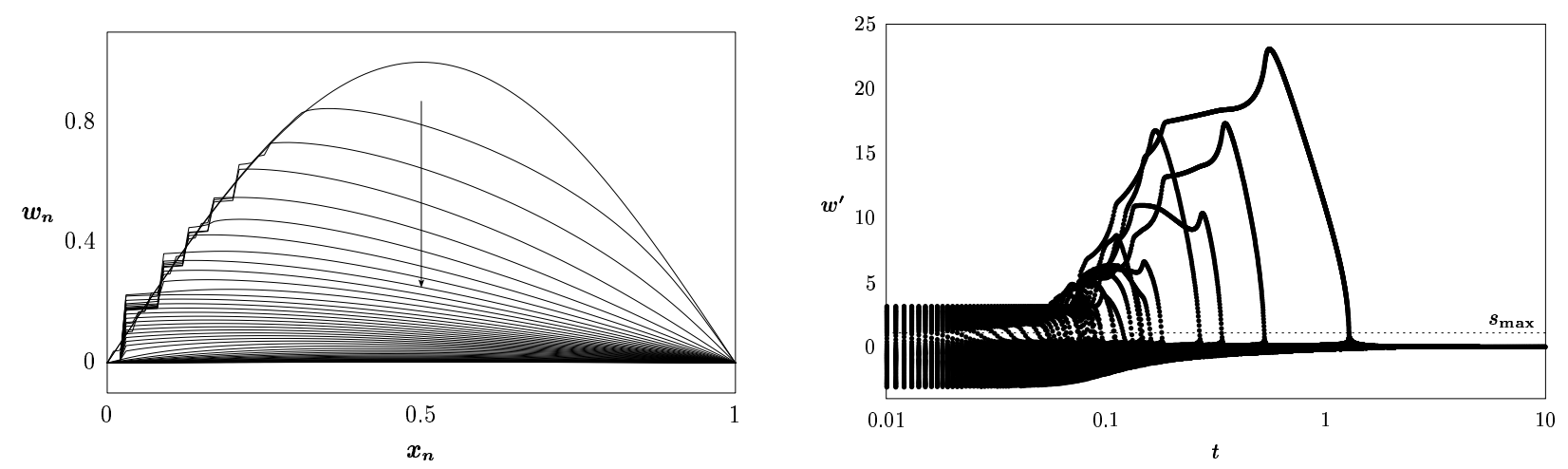

Fig. 11. Evolution for the initial value problem in Case $3^{\prime}$; (a) $w_{n}$ profiles and (b) slopes $w_{n+1 / 2}^{\prime}$ as a function of time.

As described in Proposition 3, for Case $2^{\prime}$ both the trivial solution $w=0$ and the $K=1$ strong shock solutions are stable. One way to illustrate this bi-stability is to plot the Liapunov function $\mathcal{L}$ for the one-parameter family of piecewise linear functions with $K=1$ (see Fig. 12),

$$
w_{n_{1}+1 / 2}^{\prime}=s_{+}, \quad w_{n+1 / 2}^{\prime}=-\frac{s_{+}}{N-1} \text { for } n \neq n_{1} .
$$

Then, as a function of the slope $s_{+}$at the jump, the Liapunov function (2.28) takes the form

$$
\mathcal{L}\left(s_{+}\right)=\frac{1}{N}\left[V\left(s_{+}\right)+(N-1) V\left(-\frac{s_{+}}{N-1}\right)\right] .
$$

This is a double well potential with minima corresponding to the trivial state $w=0$ and the strong shock solution (see Fig. 12a). The weak shock is an unstable equilibrium corresponding to a maximum of $\mathcal{L}$ and separates the basins of attraction of the two stable states (see Fig. 12b). While this description is quite suggestive, it is also somewhat misleading for the dynamic evolution. This is because solutions of (2.25) starting from initial data given by (5.2) do not remain within the family (5.2) with $s_{+}=s_{+}(t)$. Nevertheless, this discussion serves to point out the significance of the unstable weak shock as a boundary for the basins of attraction of the trivial and strong shock solutions. The existence of bi-stability in Case $2^{\prime}$ in a model of clustering of granular gases [35] of the same form as (2.25) with $N=3$ was 

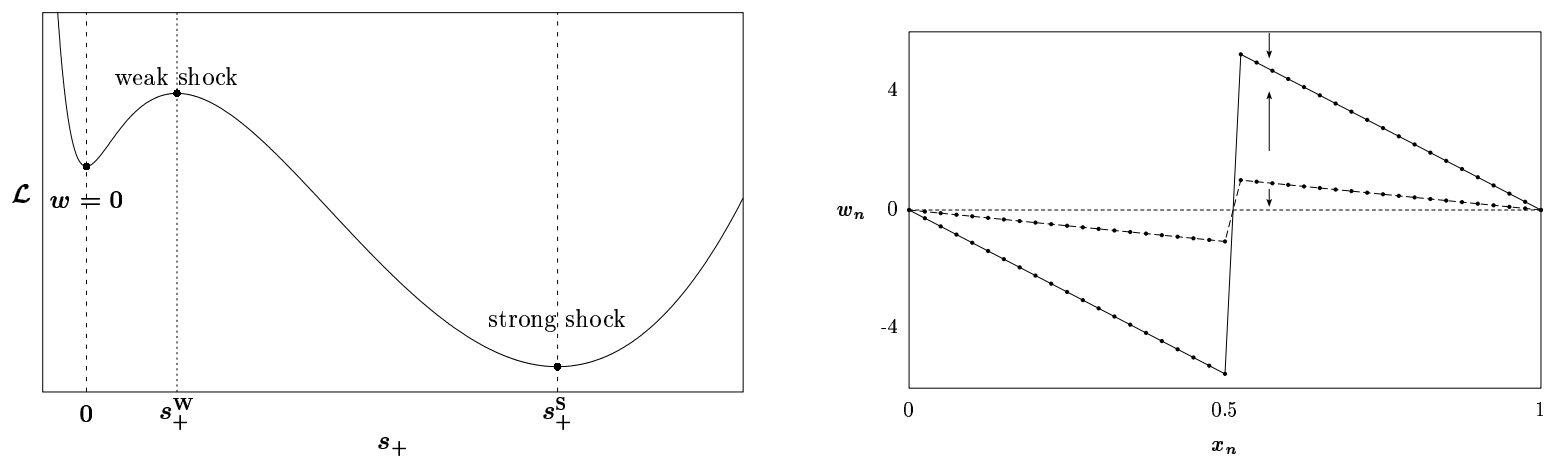

Fig. 12. (a) The double-well Liapunov function and (b) equilibria for single-jump $(K=1)$ piecewise linear solutions in Case $2^{\prime}$.

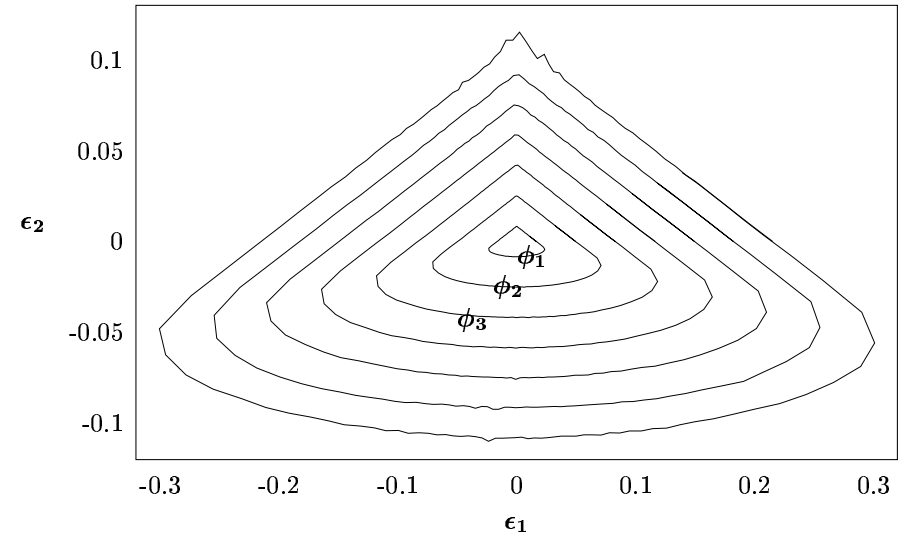

Fig. 13. The basin of attraction for the uniform state $w=0$ for $\alpha<\phi_{1}<\phi_{2}<\phi_{3}<\cdots<\beta(\alpha, N, 1)$ in Case $2^{\prime}$ given in terms of the two-parameter family of initial conditions (5.4).

studied in connection with hysteretic effects.

Next, we illustrate the dependence on the initial conditions of the large-time limit of the solution in Case 2'. Consider the discrete problem (2.25) with $N=100$ and a two-parameter family of small-amplitude initial data $\left(\epsilon_{1}, \epsilon_{2}\right.$ small $)$,

$$
w(x, 0)=\epsilon_{1} \sin (\pi x)+\epsilon_{2} \sin (2 \pi x) .
$$

In Case 2', the basin of attraction of the trivial solution depends on the value of $\phi$, with $\alpha<\phi<\beta(\alpha, N, 1)$. We plot the boundary of the basin of attraction of the trivial solution $w=0$ for various values of $\phi$ in this range, see Figure 13. All initial conditions within the basin converge to $w=0$, large-amplitude solutions outside the boundary converge to the stable strong shock solution. In the limit $\phi \rightarrow \alpha$, the basin shrinks to the origin, as in Case 1 , where $w=0$ is unstable and the shear band is the global attractor. In the limit $\phi \rightarrow \beta$, the basin of attraction for the strong shock solution shrinks to a point, where there is a saddle-node bifurcation and the weak and strong shocks coalesce (see Fig. 6). The trivial solution $w=0$ then remains as the unique (global) attractor. 

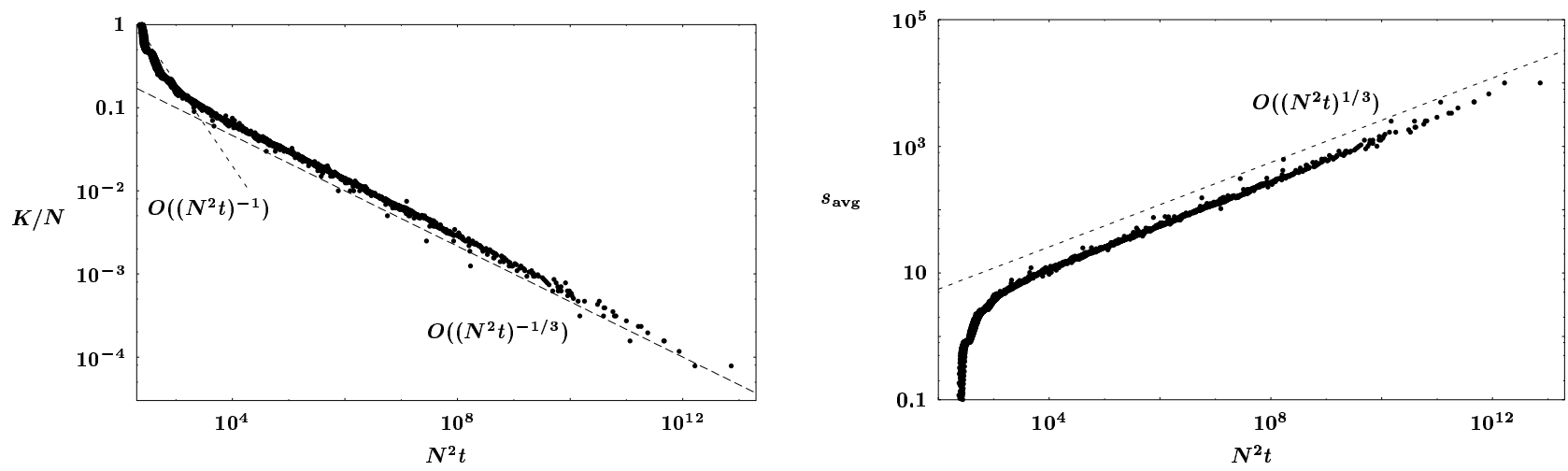

Fig. 14. Illustration of the $1 / 3$ scaling laws during the coarsening regime of evolution; (a) $K / N$, the density of jumps in the solution, $s_{+}>s_{\max }$, and (b) the slope of the average jump $s_{+}^{\text {avg }}$ for a set of simulations with a range of values for $N=100 . .25600$.

\section{Scaling laws and considerations of the continuum limit}

In this section we study in detail the coarsening dynamics of solutions of (2.25) in Case $1^{\prime}$ with initial conditions

$$
w(x, 0)=\underline{w}(x)=A \sin (\pi x),
$$

with

$$
0<|A| \ll A_{\max }
$$

where $A_{\max }=\left|s_{\max }\right| / \pi$. For this range of $A$, the condition

$$
\partial_{x} \underline{w}(x)>s_{\max },
$$

is satisfied everywhere and coarsening dynamics is observed throughout the entire interval, $0 \leq x \leq 1$. Recall from Section 2.2 that in Case 1, the trivial solution $w \equiv 0$ is linearly ill-posed and small-amplitude initial data satisfying (6.2) will be unstable everywhere in $0 \leq x \leq 1$. Grid-scale instabilities develop everywhere and evolve to a staircase profile (see Fig. 9ab) followed by coarsening dynamics, during which the jumps vanish one-by-one until just one remains. It is easier to analyze the dynamics for small initial data than the simulation shown in Fig. 9, where $A=1$. We will consider initial value problems with small initial data for Case $1^{\prime}$ of (2.25) to avoid complications that may be introduced by bi-stability in Case $2^{\prime}$ and non-uniform spatial instabilities for large amplitude data. While we show results for the specific example with initial data with $A=10^{-9}$ in Case $1^{\prime}$ with $\alpha=\pi / 8$ and $\phi=\pi / 16$, and $s_{\max } \approx-0.855$, for simulations with $N=2^{m} \times 100$ for $m=0,1,2, \ldots, 8$ grid points, simulations with other data strongly suggest that the features of the ensuing evolution are universal, for sufficiently large $N$, for all initial data satisfying (6.2).

In section 3, we defined equilibrium jumps as grid points where the discrete slope was larger than $s_{\max }, w_{n+1 / 2}^{\prime}>s_{\max }$. Here, we apply the same criterion for counting the number of jumps, $K(t)$, in an evolving solution $\left\{w_{n}(t)\right\}$. The remaining $N-K$ grid points, where 
$w_{n+1 / 2}^{\prime}<s_{\max }$, are called the background. Examination of the simulations shows that, for large times, $K(t)$, the number of finite jumps at time $t$, satisfies the scaling law

$$
K(t) \sim C\left(\frac{t}{N}\right)^{-1 / 3}
$$

for some constant $C$. A remarkable collapse (even for short times) of the data from simulations with different values of $N$ occurs if we re-express (6.3) as a scaling law for $K / N$, the density of jumps in the interval,

$$
\frac{K(t)}{N} \sim C\left(N^{2} t\right)^{-1 / 3}
$$

This scaling behavior is exhibited in Figure 14a, which contains data from simulations with $N=2^{m} \times 100$ for $m=0,1,2, \ldots, 8$ grid points.

A direct consequence of (6.4) is a scaling law for the average slope for a jump at time $t$. To see this consequence, we note that the global constraint on the slopes (3.2) holds for all times. Let $s_{+}^{\text {avg }}$ and $s_{-}^{\text {avg }}$ be the averages at time $t$ of the jump $\left(s>s_{\max }\right)$ and background $\left(s<s_{\max }\right)$ slopes respectively. Then in terms of these averages, (3.2) yields a generalization of (3.9) valid for all times,

$$
K s_{+}^{\mathrm{avg}}+(N-K) s_{-}^{\mathrm{avg}}=0 .
$$

respectively. For long times, $s_{-}^{\text {avg }} \approx s_{\text {crit }}$ and $K$ decreases, leading to $K \ll N$, so (6.5) reduces to

$$
s_{+}^{\text {avg }} \sim-\frac{N}{K} s_{\text {crit }} \sim-\frac{s_{\text {crit }}}{C}\left(N^{2} t\right)^{1 / 3}
$$

Figure 18b shows that for large times, the average slope at a jump does follow this scaling behavior.

A useful heuristic picture of the solution may be extracted from formula (6.3), considered at a fixed time $t_{0}$, as $N \rightarrow \infty$. In the coarsening dynamics regime, the solution $\left\{w_{n}\left(t_{0}\right)\right\}$ is approximately piecewise linear in $n$, with intervals of width $O\left(K^{-1}\right)=O\left(\left(t_{0} / N\right)^{1 / 3}\right)$ where the slope is close to $s_{\text {crit }}$ alternating with single grid-cells with large negative slopes of order $O\left(\left(N^{2} t_{0}\right)^{2 / 3}\right)$. As suggested by Figure 9a, wherever staircase pattern forms, it evolves so that $\left\{w_{n}\left(t_{0}\right)\right\}$ continues to follow the initial data $\underline{w}(x)$ in some approximate or locally-averaged sense. Based on this observation we introduce two norms to investigate how the solution evolves from the initial data.

Specifically, we consider two $L^{2}$ norms, for the depature of $w_{n}(t)$ from the initial condition $\underline{w}(x),(6.1)$, and for the departure of the slopes $w_{n+1 / 2}^{\prime}(t)$ from $\underline{w}_{x}(x)$,

$$
E_{w}(t, N)=\left[\sum_{n}\left|w_{n}(t)-\underline{w}(n \Delta x)\right|^{2} \Delta x\right]^{1 / 2}
$$



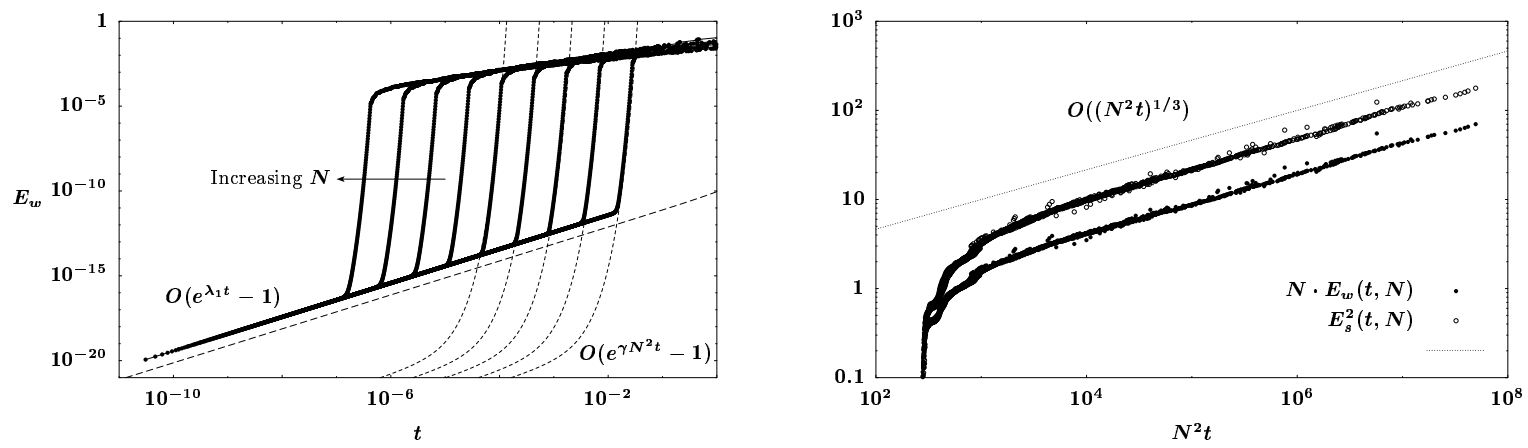

Fig. 15. Evolution of the norms, $E_{w}$ and $E_{s}$ for a series of simulations in the limit $N \rightarrow \infty$; (a) very short-time behavior showing linear growth and $N$-dependent linear instability, and (b) evolution on the fast timescale, showing saturation of the phase separation instabilities.

$$
E_{s}(t, N)=\left[\sum_{n}\left|w_{n+1 / 2}^{\prime}(t)-\underline{w}_{x}(n \Delta x)\right|^{2} \Delta x\right]^{1 / 2} .
$$

Figure 15 shows plots of these norms for a series of simulations with resolutions $N=2^{m} \times 100$ points with $m=0,1,2, \cdots, 8$. From Figure $15 \mathrm{a}$, we see that for very short times $E_{w}(t)$ shows slow exponential growth with rate $\lambda_{1} \sim-\pi^{2} F^{\prime}(0)$. This is to be expected since (6.1) is a multiple of the lowest order eigenvector of the linearization (4.5). However, since this problem is ill-posed, this smooth evolution is very quickly overwhelmed by strongly unstable high frequency modes that generate grid oscillations. These instabilities also grow exponentially, with rates on the order of the largest eigenvalue, $\lambda_{N-1}=O\left(N^{2}\right)$ as $N \rightarrow \infty$ (see Figure 15a). This instability can be regarded as the initial stage of phase separation - the formation of large gradients in the solution. Due to the maximum principle, Proposition 2, these grid oscillations cannot grow indefinitely, but saturate and lead to another stage of dynamics.

From the growth of the strong instabilities, we note that the timescale of the dynamics at the end of the regime of linearized growth is $t=O\left(N^{-2}\right)$. In fact, this timescale holds for all of the longer time dynamics of the solution. Figure 15b shows that for longer times, both norms, $E_{w}$ and $E_{s}$ depend on the rescaled time, $\tau=N^{2} t$. In fact, there is a remarkable collapse of the results from all of the simulations onto limiting curves independent of $N$ that hold after the instabilities have saturated. Figure 15b shows that for large $\tau$, the norms very closely follow the power-law scaling with exponent $1 / 3$,

$$
E_{w}(t, N) \sim \frac{C_{w}}{N}\left(N^{2} t\right)^{1 / 3}, \quad E_{s}^{2}(t, N) \sim C_{s}\left(N^{2} t\right)^{1 / 3}
$$

Changing the point of view, fixing $t$ and letting $N \rightarrow \infty$, from the scaling of $E_{s}(t)$, we note that the maximum slope in the solution will diverge like $O\left(N^{2 / 3}\right)$ (see also (6.6)), see Figure 16a. Further, note that the scalings for norms (6.9) at a fixed time simplify to

$$
E_{w}(t, N)=O\left(N^{-1 / 3}\right) \quad E_{s}^{2}(t, N)=O\left(N^{2 / 3}\right) .
$$

From this we observe: 

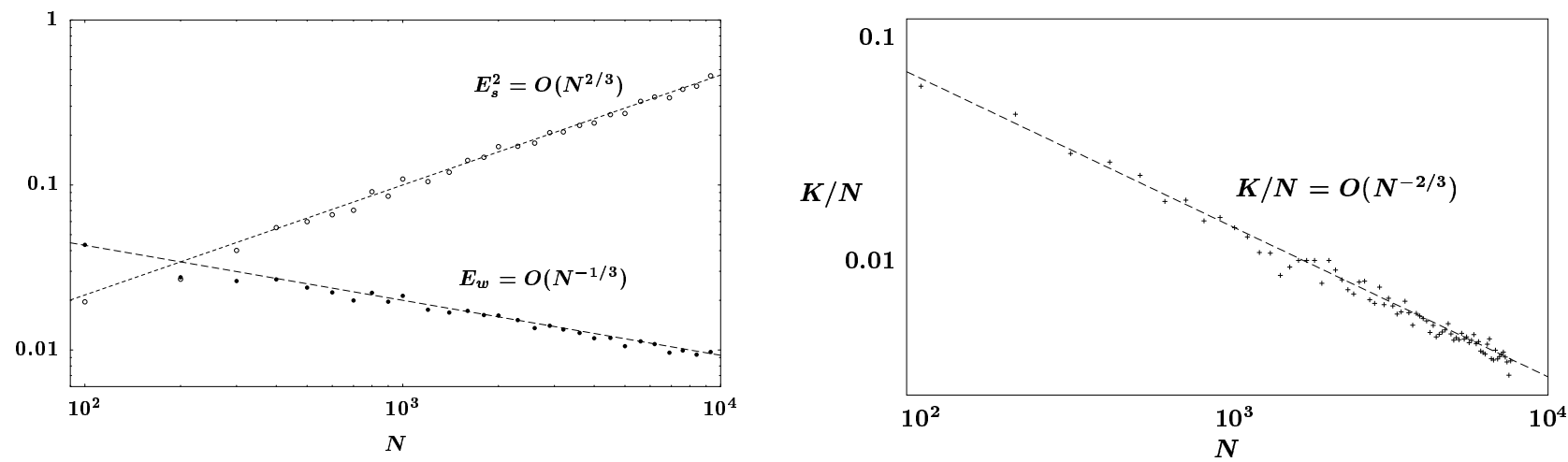

Fig. 16. Scaling laws for the properties of the solution at a fixed time, $t=1$, in the limit that $N \rightarrow \infty$; (a) the evolution norms $E_{s}^{2}=O\left(N^{2 / 3}\right)$ and $E_{w}=O\left(N^{-1 / 3}\right)$, (b) $K / N$, the density of jumps in the solution.

- For any fixed positive time $t$, as $N \rightarrow \infty$, in terms of the $L^{2}$ norm, the solution $w_{n}(t)$ will not have evolved from the initial data, since $E_{w}=O\left(N^{-1 / 3}\right) \rightarrow 0$.

- For any fixed positive time $t$, as $N \rightarrow \infty$, in terms of the $H^{1}$ norm, the solution blows up, since $E_{s}^{2}=O\left(N^{2 / 3}\right) \rightarrow \infty$.

These two observations for the finite-time behavior of the continuum limit, $N \rightarrow \infty$, describe a solution that evolves from the initial data only by instantaneously developing infinitesimally small jump discontinuities. This singular behavior for the continuum limit shows that the discreteness of model (2.25) is essential to its dynamics for any finite $N$.

Note that re-writing (6.9) for $E_{w}$ in the form

$$
E_{w}(t, N) \sim C_{w}\left(\frac{t}{N}\right)^{1 / 3}
$$

shows that (2.25) has a very long timescale, $t=O(N)$, associated with the coarsening dynamics. This is the timescale that describes the very slow overall evolution of the system. As described earlier, during coarsening, the large number of jumps initially created during the initial phase separation regime will systematically collapse producing successive solutions with fewer, larger-amplitude jumps. In the final section, we present some analysis of this dynamic behavior.

\section{Intermediate Dynamics: Coarsening}

The dynamic simulations of the previous sections suggest that while we have thoroughly studied the steady states and asymptotic stability, a complete understanding of the behavior of (2.25) requires an examination of the complicated intermediate dynamics of the system as well. For ill-posed initial data, i.e. for data with $\max \left(w_{x}\right)>s_{\max }$, the formation of a large number of jumps in the solution creates very unstable intermediate states. From Proposi- 
tion 4 , we know that there are no stable states with more than $K=1$ jumps. Consequently, the dominant feature of the evolution for all finite times will be a type of coarsening $d y$ namics - a process continually reducing the number of jumps in the solution until a stable steady state is achieved. In this section we will use two approaches to examine the dynamic behavior at a single-step transition, from $K$ to $K-1$ jumps. First, we use linear analysis to study the unstable equilibria, then we also consider an approximate reduction of the full system to a lower dimensional nonlinear system.

\subsection{Linearization at two-jump equilibria}

To assess the transient timescale for the collapse of a jump discontinuity, we estimate the positive (unstable) eigenvalue of the linearization of (2.25) at an equilibrium with two strong shocks. As was done in Section 4, the linearization can be analyzed as a perturbation of a

matrix with block structure, but now with three blocks. Specifically, if the jumps, separated by $L$ grid points, are at the grid points $n_{1}=I$ and $n_{2}=I+L$, then

$$
\mathbf{L}(\epsilon)=N^{2} F^{\prime}\left(s_{-}\right)\left(\mathbf{T}_{0}-\epsilon \mathbf{P}\right),
$$

where

$$
\mathbf{T}_{0}=\operatorname{diag}\left(\mathbf{T}_{I}^{(d n)}, \mathbf{T}_{L}^{(n n)}, \mathbf{T}_{N-I-L-1}^{(n d)}\right)
$$

$\epsilon$ is given by (4.9), and

$$
\mathbf{P}=\operatorname{diag}\left(\mathbf{0}_{I-1}, \mathbf{A}, \mathbf{0}_{L-2}, \mathbf{A}, \mathbf{0}_{N-I-L-2}\right) .
$$

Here $\mathbf{A}$ is the $2 \times 2$ matrix given by (4.11) In the following calculation we shall let $N \rightarrow \infty$ while keeping $I / N$ and $L / N$ fixed.

The matrix $\mathbf{T}_{0}$ in (7.2) is negative semidefinite with one zero eigenvalue associated with the eigenvector

$$
u=\left(0_{I}, e_{L}, 0_{N-I-L-1}\right)^{T},
$$

where $0_{I}$ denotes the $I$-dimensional zero vector and $e_{L} \in \mathbf{R}^{L}$ is given by $e_{L}=(1,1, \ldots, 1)^{T}$. This eigenvector spans the kernel of the second-difference operator with Neumann boundary conditions, $\mathbf{T}_{L}^{(n n)}$. To leading order in perturbation theory,

$$
\lambda_{\max } \sim-\epsilon N^{2} F^{\prime}\left(s_{-}\right) \frac{\langle u, \mathbf{P} u\rangle}{\langle u, u\rangle}
$$

where $\langle\cdot, \cdot \cdot\rangle$ denotes the normalized Euclidean inner product on $\mathbf{R}^{N-1},\langle u, v\rangle=\sum u_{n} v_{n} \Delta x$. It is readily computed that $\langle u, \mathbf{P} u\rangle=-2 / N$ and that $\langle u, u\rangle=L / N$; thus,

$$
\lambda_{\max } \sim \frac{2 \epsilon N^{2} F^{\prime}\left(s_{-}^{\mathrm{S}}\right)}{L} .
$$



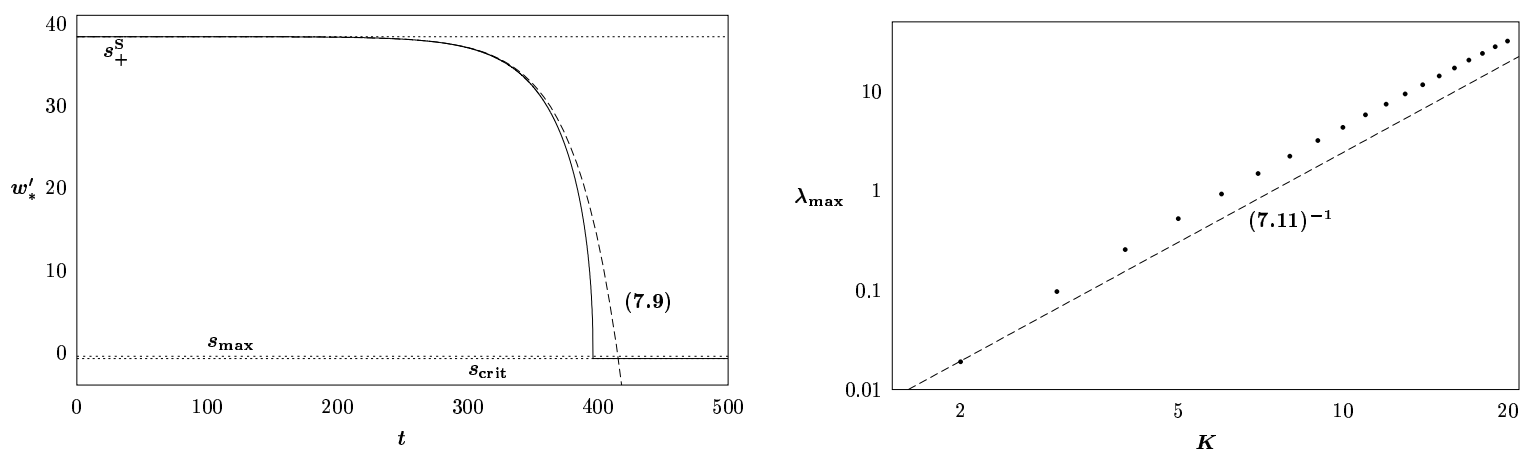

Fig. 17. Transient evolution for multiple-jump solutions; (a) time-evolution for the collapse of a jump in a $K=2$ solution (solid line) compared with the linear growth rate (7.9) (dashed line), (b) comparison of the maximum unstable eigenvalue for $K$-jump equilibria (dots) with the perturbation-theory estimate given by (7.11) (dashed line).

To estimate $\epsilon$, we use the analogue of (3.10) for a two-jump $(K=2)$ strong shock equilibrium,

$$
\begin{aligned}
& s_{-}^{\mathrm{S}}=s_{\text {crit }}+O\left(N^{-1}\right)<0, \\
& s_{+}^{\mathrm{S}}=-(N-2) s_{\text {crit }} / 2+O(1)>0 .
\end{aligned}
$$

Using (4.9) and (7.7), we obtain the value of $\epsilon$ for the two-jump strong shock solution in terms of the previous result (4.12),

$$
\epsilon=\epsilon_{2}^{\mathrm{S}}=-\frac{F^{\prime}\left(s_{+}^{\mathrm{S}}\right)}{F^{\prime}\left(s_{-}^{\mathrm{S}}\right)} \sim 4 \epsilon_{1}^{\mathrm{S}}=O\left(N^{-2}\right),
$$

Consequently, we obtain the estimate ${ }^{2}$

$$
\lambda_{\max } \sim \frac{8 \sin \alpha \sin \phi}{L s_{\text {crit }}^{2}} .
$$

Of course $1 / \lambda_{\max }$ defines the time scale for the collapse of a two-shock meta-stable equilibrium; in particular, since $L$ scales like $N / K$, the collapse time is proportional to $N$ as $N \rightarrow \infty$. Figure 17a illustrates the accuracy of this linear estimate for the evolution for the collapsing jump, starting from a small perturbation of the $K=2$ strong equilibrium (7.7), see Figure 7a. The linear growth rate (7.9) gives a very good estimate of the evolution until the jump has almost completely collapsed, $w_{n_{1}+1 / 2}^{\prime} \sim s_{\max }$.

More generally, this argument can be extended to show that if there are $K$ jumps at points $n_{1}, n_{2}, \ldots, n_{K}$ where $K \ll N$, then the perturbation-theory estimate for the largest positive

$\overline{2}$ Note that, like $\epsilon$, the smallest eigenvalues of $\mathbf{T}_{0}$ are $O\left(N^{-2}\right)$. As a check on the accuracy of applying perturbation theory when the perturbations are of the same order as the eigenvalues, we showed that the second order correction to the eigenvalue is $O\left(\epsilon N^{-2}\right)$, smaller than the first order correction by a factor of $N$. Moreover, we determined the lowest eigenvalue of $\mathbf{T}_{0}-\epsilon \mathbf{P}$ using Sturm sequences (see [16]), and this yielded the same results as perturbation theory. 
eigenvalue of the linearization is

$$
\lambda_{\max } \sim \frac{2 \epsilon_{K}^{\mathrm{S}} N^{2} F^{\prime}\left(s_{-}^{\mathrm{S}}\right)}{\min _{k}\left(n_{k}-n_{k-1}\right)} .
$$

If the jumps are approximately equally spaced, then the denominator is $L_{\min } \sim N / K$. For solutions with $K$ jumps, $\epsilon_{K}^{\mathrm{S}} \sim K^{2} \epsilon_{1}^{\mathrm{S}}$, where $\epsilon_{1}^{\mathrm{S}}=O\left(N^{-2}\right)$, see (4.12). Consequently, our crude estimate for the timescale for the transition from $K$ to $K-1$ jumps is

$$
\frac{1}{\lambda_{\max }} \sim \frac{s_{\mathrm{crit}}^{2}}{2 \sin \alpha \sin \phi} \frac{N}{K^{3}}
$$

A comparison of this estimate of the maximum growth rate with the largest unstable eigenvalue of the $K$-jump strong equilibria is shown in Figure $17 \mathrm{~b}$, which confirms that $\lambda_{\max }=O\left(K^{3}\right)$. For $K \geq 2$, the strong shock equilibria have $K-1$ closely spaced positive eigenvalues, and a simplified single-mode linearized analysis may be insufficient to describe the dynamic evolution of the problem for moderate to larger values of $K$.

We note that the results of the linearized analysis, (7.11), suggest that the scaling law for $K(t)$ should have the exponent one half rather than the actual value of one third, observed from the simulations in Section 6. The failure of this linear estimate indicates that the dynamic solution $w_{n}(t)$ does not come arbitrarily close to the unstable $K$-jump equilibria during the coarsening process. In consequence, the rate of collapse of the jumps is faster than the estimate from linear theory.

\subsection{The jump-diffusion model}

In this subsection, we formulate a model that seeks to isolate the evolution of the finite jumps, the main feature of the nonlinear coarsening dynamics. Let $s_{n}$ denote the discrete slope

$$
s_{n} \equiv w_{n+1 / 2}^{\prime}=\frac{w_{n+1}-w_{n}}{\Delta x}, \quad n=0,1,2, \cdots, N-1 .
$$

Taking differences of (2.25), we write the equivalent slope-evolution equations,

$$
\frac{d s_{n}}{d t}=\frac{F\left(s_{n+1}\right)-2 F\left(s_{n}\right)+F\left(s_{n-1}\right)}{\Delta x^{2}}, \quad 1 \leq n \leq N-2 .
$$

Similarly, at the edges of the domain, $n=0$ and $n=N-1$,

$$
\frac{d s_{0}}{d t}=\frac{F\left(s_{1}\right)-F\left(s_{0}\right)}{\Delta x^{2}}, \quad \frac{d s_{N-1}}{d t}=-\frac{F\left(s_{N-1}\right)-F\left(s_{N-2}\right)}{\Delta x^{2}} .
$$

Equations (7.14) are derived from the Dirichlet boundary conditions (2.26). In particular, the equation for $s_{N-1}$ is obtained by differentiating the boundary condition constraint (3.2), 
in the form

$$
s_{N-1}=-\sum_{n=0}^{N-2} s_{n}
$$

with respect to $t$ and using (7.13) to collapse the sum.

Note that equations (7.13), are a second-order accurate finite difference discretization of the slope diffusion PDE (2.17):

$$
\frac{\partial s}{\partial t}=\frac{\partial^{2}}{\partial x^{2}}(F(s))
$$

The jump in $w_{n}$ is expressed by

$$
w_{n+1}-w_{n}=s_{n} \Delta x
$$

This quantity corresponds to a jump in $w$ as $\Delta x \rightarrow 0$ only when $s_{n}$ is large, $s_{n}=O(N)$ as $N \rightarrow \infty$. In the intermediate dynamics, we find that only large positive values of $s_{n}$ can occur, with $s_{n}>s_{\max }$, as was the case for the stable equilibrium solutions found in section 3 . Consequently, as was done in Section 6 , points in the solution $\left\{w_{n}\right\}$ where the slope satisfies $s_{n}>s_{\max }$ will be referred to as jumps, and the remaining points with small slopes, $s_{n} \leq s_{\max }$, will be called the background.

In Figure 18a, we show a typical numerical simulation with $N=500$, at a time when there are $K=20$ jumps on $0<x<1$. The evolution of a jump is controlled by the second difference $\delta^{2} F\left(s_{n}\right) \equiv F\left(s_{n+1}\right)-2 F\left(s_{n}\right)+F\left(s_{n-1}\right)$, which appears as the numerator of (7.14). Specifically, whether a jump will grow or decay depends on if $\delta^{2} F\left(s_{n}\right)$ is positive or negative, respectively. Figure 18a shows a portion of the solution $w_{n}, 0 \leq n<180$, containing seven jumps (label them $\sigma_{1}, \sigma_{2}, \cdots, \sigma_{7}$ ) and Figure $18 \mathrm{~b}$ shows the corresponding flux $F\left(s_{n}\right)$. Note that the flux is continuous everywhere, and the first difference, $\delta F\left(s_{n}\right) \equiv F\left(s_{n+1}\right)-F\left(s_{n}\right)$, is piecewise constant with jumps corresponding to those of $w_{n}$. Moreover, Figure 18b shows that the second difference $\delta^{2} F$ is negative for jumps $\sigma_{2}$ and $\sigma_{6}$, hence those jumps will decay in amplitude. Also note that the jump labelled $\sigma_{4}$ is close to equilibrium, since locally $F\left(s_{n}\right)$ is nearly linear and hence $\delta^{2} F\left(\sigma_{4}\right)$ is near zero (see Figure 18b).

Consider a solution of $(7.13,7.14)$ starting with $K$ interior jumps at some initial time. Numerical simulations suggest that the background, with $s_{n}<s_{\max }$, equilibrates on a fast time-scale, and thereafter evolves quasi-statically, being driven by the slower evolution of the jumps. In particular, since the background is at quasi-static equilibrium, we deduce that $F\left(s_{n}\right)$ must be approximately linear between jumps, as indicated in Figure 18b. We further observe in this figure that $F\left(s_{n}\right)$ appears continuous at the jumps. Consequently, the flux of solution, $F\left(s_{n}\right)$, is determined everywhere by the values of $F\left(s_{n}\right)$ at the jumps alone. With these observations, we can formulate a closed system of equations for the evolution of the jumps that is decoupled from the quasi-static evolution of the background. 
Let $\sigma_{k}=s_{n_{k}}$ denote the slope at the $k^{\text {th }}$ jump. Taking $F\left(s_{n}\right)$ to be linear in $n$ between jumps yields the model

$$
F\left(s_{n}\right)=F\left(\sigma_{k}\right)+\frac{F\left(\sigma_{k+1}\right)-F\left(\sigma_{k}\right)}{n_{k+1}-n_{k}}\left(n-n_{k}\right) \quad \text { for } n_{k} \leq n \leq n_{k+1} .
$$

To specify this model for the flux to the left of the first jump, and to the right of the $K^{\text {th }}$ jump, define $\sigma_{0}=s_{0}$ and $\sigma_{K+1}=s_{N-1}$. Then $F\left(s_{n}\right)$ is defined for all $0 \leq n \leq N-1$ by (7.18) in terms of $\sigma_{k}$ for $0 \leq k \leq K$. Since the jumps are assumed to be interior, both $s_{0}$ and $s_{N-1}$ are less than $s_{\max }$, are part of the background, and hence evolve quasi-statically. In particular, the rates of evolution for $\sigma_{0}$ and $\sigma_{K+1}$ in (7.14) are of lower order than those of the rates of evolution for the jumps $\left\{\sigma_{k}\right\}$. Balancing terms in (7.14) forces the conditions that $F\left(s_{1}\right)=F\left(s_{0}\right)$ and $F\left(s_{N-2}\right)=F\left(s_{N-1}\right)$. Consequently, the slope of $F\left(s_{n}\right)$ adjacent to each boundary is zero, and hence

$$
F\left(\sigma_{0}\right)=F\left(\sigma_{1}\right), \quad F\left(\sigma_{K+1}\right)=F\left(\sigma_{K}\right) .
$$

After some manipulation, substitution of (7.18) into (7.20) leads to the equations

$$
\frac{d \sigma_{k}}{d t}=\frac{1}{\Delta x^{2}}\left(\frac{F\left(\sigma_{k+1}\right)-F\left(\sigma_{k}\right)}{n_{k+1}-n_{k}}-\frac{F\left(\sigma_{k}\right)-F\left(\sigma_{k-1}\right)}{n_{k}-n_{k-1}}\right), \quad 2 \leq k \leq K-1
$$

and similarly including (7.19) gives equations for $\sigma_{1}$ and $\sigma_{K}$ :

$$
\frac{d \sigma_{1}}{d t}=\frac{F\left(\sigma_{2}\right)-F\left(\sigma_{1}\right)}{\left(n_{2}-n_{1}\right) \Delta x^{2}}, \quad \frac{d \sigma_{K}}{d t}=-\frac{F\left(\sigma_{K}\right)-F\left(\sigma_{K-1}\right)}{\left(n_{K}-n_{K-1}\right) \Delta x^{2}} .
$$

This system is similar in form to (7.14), but it represents a vast reduction of the problem when $K \ll N$ - we have to consider only $K$ coupled equations at the jumps, rather than $N$ equations at all of the points in the domain. Whereas (7.13) describes a finite difference scheme for (7.16) on a uniform grid, (7.20) is a discretization of (7.16) on a non-uniform grid, given by the positions of the jumps, $\left\{n_{k}\right\}$.

It is perhaps worth considering the evolution of jumps in the context of the nonlinear diffusion PDE (7.16) for the slope field $s(x, t)$. If the solution $w(x, t)$ of $(2.10)$ contains a finite number of jump discontinuities at locations $x_{k}$, then the slope, $s \equiv \partial_{x} w$, is a distribution containing delta-functions at $x_{k}$. Assuming the jump locations $x_{k}$ are stationary (independent of $t$ ), we find that $\partial_{t} s$ also has delta functions at the jumps. Consequently, interpreting (7.16) in the sense of distributions, we find that the flux $F(s)$ is continuous in space and $\partial_{x} F(s)$ is piecewise continuous in space with jumps at $x_{k}$. Moreover, at the jumps, we have

$$
\partial_{t}[w]_{k}=\left[\partial_{x} F(s)\right]_{k}
$$

where $[w]_{k} \equiv w\left(x_{k}^{+}, t\right)-w\left(x_{k}^{-}, t\right)$ denotes the $k^{\text {th }}$ jump in $w$, and the right hand side denotes the corresponding jump in $\partial_{x} F(s)$. Thus, in the continuum version of the jump-diffusion model $(7.20)$, it is not clear how to express $[w]_{k}$ in terms of $s$, so the system is not closed. 

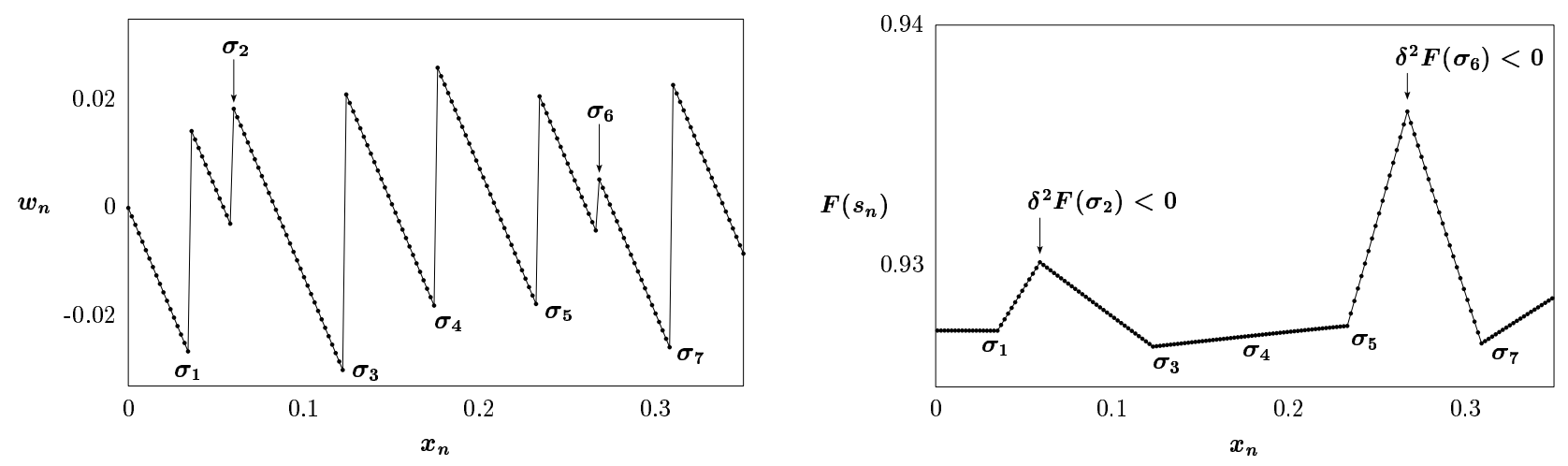

Fig. 18. (left) A portion of a solution $\left\{w_{n}\left(t_{0}\right)\right\}$ with $N=500$ containing $K=20$ jumps, seven of which are on $0 \leq x<0.35$. (right) A plot of the corresponding values of the flux function, $F\left(s_{n}\right)$ indicating the decaying jumps, where $\delta^{2} F(s)<0$.

This is in contrast with the discrete model, for which jumps in $w_{n}$ are related to $s_{n}$ through equation (7.17).

We now justify the claim of separation of time-scales in the discrete model when $K \ll N$. As described earlier, if the slopes $\left\{s_{n}\right\}$ for the background correspond to a smooth function $s(x, t)<s_{\max }$, then as $N \rightarrow \infty$, then (7.13) converges to the nonlinear forward-diffusion equation (7.16) with time-scales independent of $N$. Hence $t=O(1)$ for the evolution of transients in the background. In contrast, for large $N$, the jumps are $\sigma_{k}=O(N)$, and by using the asymptotics of the flux for large $s \rightarrow \infty,(2.15)$, in (7.20) yields the slow time-scale for the evolution of the jumps as $t=O(N)$. This observation was obtained in section 6.1 for the decay rate of unstable jumps in the near-equilibrium case, (7.11).

We now briefly review the details of what happens as a jump collapses. Decay of a jump occurs on the $O(N)$ long time-scale if $\delta^{2} F\left(\sigma_{k}\right)<0$; the slope decreases to a value with $\sigma_{k}<s_{\max }$. Local equilibrium will be achieved when $\delta^{2} F\left(\sigma_{k}\right)=0$. As the evolution proceeds, the diffusion coefficient, $D\left(\sigma_{k}\right)=F^{\prime}\left(\sigma_{k}\right)$ changes sign from negative to positive, as $\sigma_{k}$ decreases through $s_{\max }$. Consequently, further local evolution resembles that of a forward parabolic equation, serving to smooth out gradients to the background, $s_{n} \sim s_{\text {crit }}$ on the fast $O(1)$ time scale. Moreover, when a jump $\sigma_{k}$ collapses, then that grid point $n_{k}$ becomes part of the background, and the system $(7.20,7.21)$ is reduced to a $(K-1)$-dimensional system for the remaining jumps. This model of the piecewise-in-time evolution of system (2.25) is supported by Figures 10, 11 and 17a, which show piecewise smooth dynamics punctuated by the collapses of jumps at finite times. It is also appropriate to note that the reduced model (7.20) gives numerical results that are indistinguishable from simulations of the full discrete model (7.13), apart from short $O(1)$ transients associated with jump collapse in the regime $s_{\text {crit }}<\sigma_{k}<s_{\max }$.

The finite-time collapse of unstable jumps typically occurs one jump at a time, (see Figure 10b). In a further simplification of the reduced model, we show how to isolate the evolution of a single jump, and show that the simplification leads to only small inaccuracies in the simulation, and the possibility of increased understanding of the collapse mechanism. 

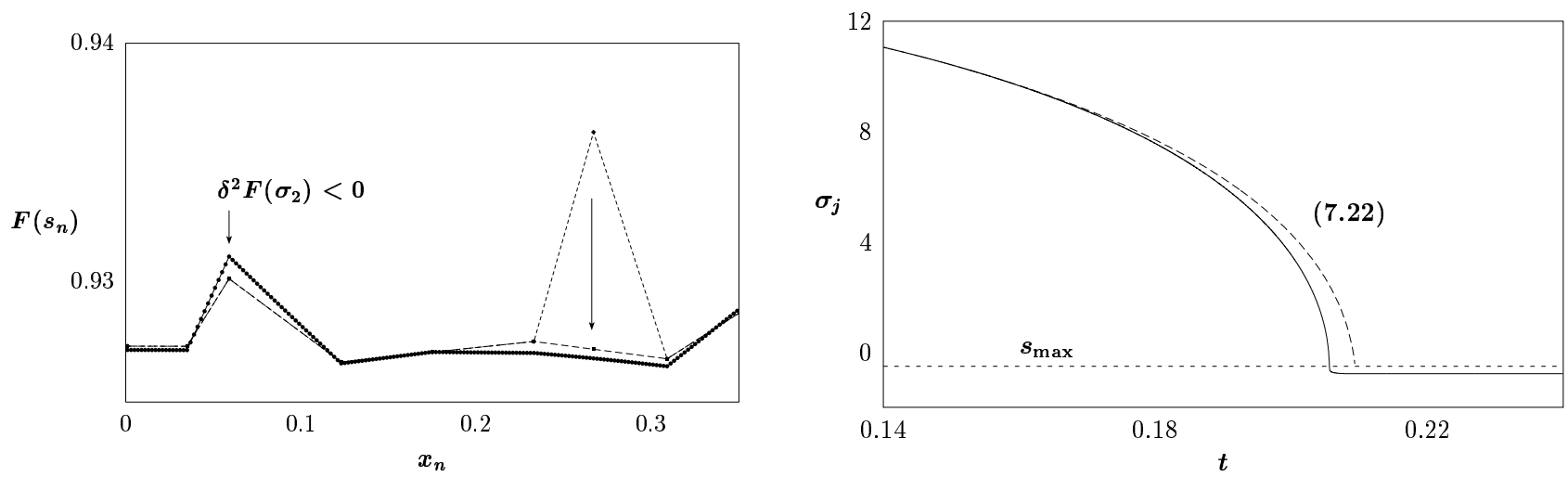

Fig. 19. (left) Comparison of the piecewise linear flux $F(s),(7.18)$, predicted by (7.22) after the collapse of jump $\sigma_{6}$ with the actual solution. (right) Comparison of the decay of jump $\sigma_{6}$ predicted by (7.22) with the actual evolution.

Let us assume that in the coarsening process, there is a separation in the timescales of the successive collapses of the jumps $\left\{\sigma_{k}\right\}$. This assumption is valid if there is a separation in the values of $\delta^{2} F\left(\sigma_{k}\right)$ for the jumps $\sigma_{k}$, i.e. if $-\delta^{2} F\left(\sigma_{1}\right) \approx-\delta^{2} F\left(\sigma_{2}\right) \approx-\delta^{2} F\left(\sigma_{3}\right) \cdots \ll-\delta^{2} F\left(\sigma_{j}\right)$ then $\sigma_{j}$ will be the next jump to collapse; this is the case for $\sigma_{6}$ in Figure 18b. Then during the finite time that the jump $\sigma_{j}$ collapses to $\sigma_{j} \rightarrow s_{\max }$, the remaining jumps will have changed only slightly (see Figure 19a). Consequently, if we neglect the slow evolution of the other jumps $s_{k}$, then system (7.20) reduces to a single first-order ODE for $\sigma_{j}$ while all of the other $\sigma_{k}$ are held constant,

$$
\frac{d \sigma_{j}}{d t}=\frac{A_{j}-B_{j} F\left(\sigma_{j}\right)}{\Delta x^{2}}
$$

where the constants $A_{j}, B_{j}$ are given by

$$
A_{j}=\frac{F\left(\sigma_{j+1}\right)}{n_{j+1}-n_{j}}+\frac{F\left(\sigma_{j-1}\right)}{n_{j}-n_{j-1}} \quad B_{j}=\frac{n_{j+1}-n_{j-1}}{\left(n_{j+1}-n_{j}\right)\left(n_{j}-n_{j-1}\right)} .
$$

Equation (7.22) may be integrated starting from the initial size of slope $s_{j}$ at time $T_{K}$, when the solution has $K$ jumps, to determine time $T_{K-1}$, when collapse has occurred, $\sigma_{j}=s_{\max }$, leaving a solution with $K-1$ jump discontinuities. If further we assume that all of the jumps are equally spaced, with $\left(n_{j+1}-n_{j}\right) \Delta x=1 / K$ and the jumps neighboring $\sigma_{j}$ are very near equilibrium, $F\left(\sigma_{j-1}\right)=F\left(\sigma_{j+1}\right)=F\left(s_{\text {crit }}\right)$, then $(7.22)$ becomes

$$
\frac{d \sigma_{j}}{d t} \approx \frac{2 K}{\Delta x}\left(F\left(s_{\text {crit }}\right)-F\left(\sigma_{j}\right)\right) \sim-\frac{2 N K \sin \alpha \sin \phi}{\sigma_{j}}
$$

where the second approximation results from the asymptotics of $F(s)$ for large $s,(2.15)$. This equation has the approximate solution

$$
\sigma_{j}(t) \sim \sqrt{4 N K \sin \alpha \sin \phi\left(T_{K-1}-t\right)}, \quad T_{K} \leq t<T_{K-1},
$$

where $T_{K-1}$ is the collapse time. From Figure 19b, we see that (7.25) qualitatively captures the nature of the finite time collapse of the jump. Figure 19 illustrates the use of the piecewise 
linear flux approximation (7.18) and equation (7.22) to calculate the collapse of the $\sigma_{6}$ jump starting from the initial conditions given in Figure 18.

This simplified model (7.22) can be expected to approximately describe the dynamics in some intermediate regime, $1 \ll K \ll N$. As long as $K \ll N$, the piecewise linear approximation (7.18) will describe the flux, but as $K \rightarrow 1$, (7.22) can not hold, because there will be strong coupling between jumps due to the boundary condition constraint (7.15). In practice, we have observed that the long-term dynamics are very sensitive to the spatial coupling of the jumps. A scaling law is observed for the number of jumps $K$ as a function of time for systems with large $N$. Equation (7.22) does not capture this, but the jump diffusion model (7.20, 7.21) does reproduce this behavior of the full system (2.25).

If we use the results of the reduced jump-diffusion model $(7.22,7.25)$ with the initial values for the jump sizes obtained from the numerical simulations from Section $6, \sigma_{j}\left(T_{K-1}\right)=$ $O\left(N / K^{3 / 2}\right)$. Then we obtain that the transition time from $K$ to $K-1$ jumps is

$$
\Delta T_{K}=O\left(N / K^{4}\right)
$$

Consequently, the cumulative time until only $K$ jumps remain is given by the summation

$$
T_{K}=\sum_{k=N}^{K} \Delta T_{k} \sim O(N) \sum_{k=N}^{K} k^{-4}=O\left(N / K^{3}\right),
$$

where the second summation can be expressed exactly in terms of the polygamma function [1] as $\sum k^{-3}=\left(\psi^{(3)}(N)-\psi^{(3)}(K+1)\right) / 6 \sim K^{-3} / 3+O\left(N^{-3}, K^{-4}\right)$. This result agrees with the estimate $K=O\left((N / t)^{1 / 3}\right)$ from (6.3). It is not clear how to derive the scaling result for $\sigma_{j}\left(T_{K-1}\right)$ from $(7.22)$ or $(7.20)$.

In conclusion, we have shown that in the continuum limit, the slow timescale for evolution in (2.25) diverges as the microscopic discretization lengthscale vanishes, $\Delta x=N^{-1} \rightarrow 0$. This singular behavior is a consequence of the asymptotic form of the non-monotone flux function, $F(s)$ for $s \rightarrow \infty$. Further work focusing on the influence of different forms of the flux function $F(s)$ is being pursued.

\section{Acknowledgments}

DGS was supported by NSF grant DMS 9803305. MS was supported by ARO grant DAAG5598-1-0128 and NSF grants DMS 9818900 and DMS 0073841. TPW was supported by an Alfred P. Sloan foundation fellowship. We wish to thank D. Lohse for sharing a preprint of his group's work with us. 


\section{References}

[1] M. Abramowitz and I. A. Stegun, editors. Handbook of mathematical functions with formulas, graphs, and mathematical tables. Dover Publications Inc., New York, 1992. Reprint of the 1972 edition.

[2] L. Alvarez, P.-L. Lions, and J.-M. Morel. Image selective smoothing and edge detection by nonlinear diffusion. II. SIAM J. Numer. Anal., 29(3):845-866, 1992.

[3] L. J. An and A. Peirce. The effect of microstructure on elastic-plastic models. SIAM J. Appl. Math., 54(3):708-730, 1994.

[4] L. J. An and A. Peirce. A weakly nonlinear analysis of elastoplastic-microstructure models. SIAM J. Appl. Math., 55(1):136-155, 1995.

[5] F. Andreu, V. Caselles, J. I. Diaz, and J. M. Mazon. Some qualitative properties for the total variational flow. IMA preprint, 1738, 2000.

[6] S. G. Bardenhagen, J. U. Brackbill, and D. Sulsky. Numerical study of stress distribution in sheared granular material in two dimensions. Phys. Rev. E, 62(3):3882-3890, 2000.

[7] J. W. Cahn. Spinodal decomposition. Trans. Metal. Soc. AIME, 242:166-180, 1968.

[8] F. Catté, P.-L. Lions, J.-M. Morel, and T. Coll. Image selective smoothing and edge detection by nonlinear diffusion. SIAM J. Numer. Anal., 29(1):182-193, 1992.

[9] P. G. de Gennes. Granular matter: a tentative view. Rev. Mod. Phys., 71(2):S374-S382, 1999.

[10] J. Eggers. Sand as Maxwell's demon. Phys. Rev. Let., 83(25):5322-5325, 1999.

[11] L. C. Evans and J. Spruck. Motion of level sets by mean curvature. I. J. Differential Geom., 33(3):635-681, 1991.

[12] F. X. Garaizar. Numerical computations for antiplane shear in a granular flow model. Quart. Appl. Math., 52(2):289-309, 1994.

[13] F. X. Garaizar and D. G. Schaeffer. Numerical computations for shear bands in an antiplane shear model. J. Mech. Phys. Solids, 42(1):21-50, 1994.

[14] K. Höllig and J. A. Nohel. A diffusion equation with a nonmonotone constitutive function. In Systems of nonlinear partial differential equations (Oxford, 1982), pages 409-422. Reidel, Dordrecht, 1983.

[15] J. M. Hyman, B. Nicolaenko, and S. Zaleski. Order and complexity in the KuramotoSivashinsky model of weakly turbulent interfaces. Phys. D, 23(1-3):265-292, 1986. Spatiotemporal coherence and chaos in physical systems (Los Alamos, N.M., 1986).

[16] E. Isaacson and H. B. Keller. Analysis of numerical methods. Dover Publications Inc., New York, 1994.

[17] A. Iserles. A first course in the numerical analysis of differential equations. Cambridge University Press, Cambridge, 1996. 
[18] F. John. Partial differential equations. Springer-Verlag, New York, fourth edition, 1991.

[19] L. P. Kadanoff. Built upon sand: Theoretical ideas inspired by granular flows. Rev. Mod. Phys., 71(1):435-443, 1999.

[20] S. Kichenassamy. The Perona-Malik paradox. SIAM J. Appl. Math., 57(5):1328-1342, 1997.

[21] M. Lizana and V. Padron. A spatially discrete model for aggregating populations. J. Math. Biol., 38(1):79-102, 1999.

[22] R.M. Neddermann. Statics and Kinematics of Granular Materials. Cambridge Univ. Press, Cambridge, 1992.

[23] V. Padron. Sobolev regularization of a nonlinear ill-posed parabolic problem as a model for aggregating populations. Comm. Partial Differential Equations, 23(3-4):457-486, 1998.

[24] P. Perona and J. Malik. Scale-space and edge detection using anisotropic diffusion. IEEE Trans. Pattern Anal. Machine Intell., 12:629-639, 1990.

[25] E. B. Pitman and D. G. Schaeffer. Instability and ill-posedness in granular flow. In Current progress in hyberbolic systems: Riemann problems and computations (Brunswick, ME, 1988), pages 241-250. Amer. Math. Soc., Providence, RI, 1989.

[26] P. Rosenau. Dynamics of nonlinear mass-spring chains near the continuum limit. Phys. Lett. $A, 118(5): 222-227,1986$.

[27] P. Rosenau. Dynamics of dense lattices. Phys. Rev. B (3), 36(11):5868-5876, 1987.

[28] P. Rosenau. Quasicontinuous spatial motion of a mass-spring chain. Phys. D, 27(1-2):224-234, 1987.

[29] J. Rubinstein and P. Sternberg. Nonlocal reaction-diffusion equations and nucleation. IMA J. Appl. Math., 48(3):249-264, 1992.

[30] J. Rubinstein, P. Sternberg, and J. B. Keller. Fast reaction, slow diffusion, and curve shortening. SIAM J. Appl. Math., 49(1):116-133, 1989.

[31] D. G. Schaeffer. Instability in the evolution equations describing incompressible granular flow. J. Differential Equations, 66(1):19-50, 1987.

[32] D. G. Schaeffer. Instability and ill-posedness in the deformation of granular materials. Internat. J. Numer. Anal. Methods Geomech., 14(4):253-278, 1990.

[33] D. G. Schaeffer. A mathematical model for localization in granular flow. Proc. Roy. Soc. London Ser. A, 436(1897):217-250, 1992.

[34] D. G. Schaeffer, M. Shearer, and E. B. Pitman. Instability in critical state theories of granular flow. SIAM J. Appl. Math., 50(1):33-47, 1990.

[35] K. van der Weele, D. van der Meer, M. Versluis, and D. Lohse. Hysteretic clustering in granular gas. preprint, 2000. 